\title{
Compact Matrix Pseudogroups
}

\author{
S. L. Woronowicz \\ Department of Mathematical Methods of Physics, Faculty of Physics, University of Warsaw, \\ Hoza 74, PL-00-682 Warszawa, Poland
}

\begin{abstract}
The compact matrix pseudogroup is a non-commutative compact space endowed with a group structure. The precise definition is given and a number of examples is presented. Among them we have compact group of matrices, duals of discrete groups and twisted (deformed) $S U(N)$ groups. The representation theory is developed. It turns out that the tensor product of representations depends essentially on their order. The existence and the uniqueness of the Haar measure is proved and the orthonormality relations for matrix elements of irreducible representations are derived. The form of these relations differs from that in the group case. This is due to the fact that the Haar measure on pseudogroups is not central in general. The corresponding modular properties are discussed. The Haar measures on the twisted $S U(2)$ group and on the finite matrix pseudogroup are found.
\end{abstract}

\section{Introduction}

Let $G$ be a Lie group. A family $\left(G_{\tau}\right)_{\tau \in[0, \varepsilon[}$ of Lie groups is said to be a deformation of $G$ if $G_{0}=G$ and $G_{\tau}$ depends continuously on $\tau$. The latter should be understood in a natural sense. For example one may require that all $G_{\tau}$ are of the same dimensions and that it is possible to choose bases in $g_{\tau}\left(g_{\tau}\right.$ is the Lie algebra of $\left.G_{\tau}\right)$ such that the corresponding structure constants depend continuously on $\tau$.

Assume that the group $G$ is involved in a theory (e.g. it is a symmetry group) describing a physical reality. As we well know any physical theory describes well only a limited class of phenomena, for the phenomena beyond this class the theoretical predictions disagree with the experimental results. In order to obtain the adequate description of a larger class of phenomena one must modify the theory. In certain cases such a modification although revolutionary from the conceptual point of view consists in replacing $G$ by one of the group $G_{\tau}$. Then the value of $\tau$ is one of the fundamental constants (small parameter) of the new, more general theory. Within this new theory the group $G$ retains its validity only in the approximate sense (e.g. it describes a broken symmetry). The old theory can be recovered in the limit $\tau \rightarrow 0$. 
The history of physics provides many examples of developments that fit into the scheme described above. Births of the special theory of relativity and the theory of quanta are the most famous. Another example we get by considering the symmetry group of the flat Minkowski space-time of special theory of relativity, i.e. the Poincaré group. It admits a non-trivial deformation that leads to the theory of the de Sitter space-time.

The above consideration indicate that studying all possible deformations of a group involved in a physical theory, one may discover ways leading to more general theories that might better describe the reality.

This procedure seems to be especially useful if already in the existing theory it is known that the symmetry described by the considered group is broken. Such situations are constantly met in elementary particle physics where we mainly deal with compact semisimple Lie groups. Unfortunately these groups are rigid: they admit only trivial deformations (a deformation $\left(G_{\tau}\right)_{\tau \in[0, \varepsilon[}$ is said to be trivial if all $G_{\tau}$ are isomorphic to $G$ ). If however we extend the notion of compact group including non-commutative compact spaces (compact pseudospaces in the sense of [17]) endowed with a group structure, then the class of deformations becomes richer and one can find non-trivial deformations for symmetry groups in elementary particle physics. For $S U(2)$ such a deformation is described in [18].

Let $A$ be a $C^{*}$-algebra with unity. If $A$ is commutative, then according to the Gelfand-Naimark theory $A$ is isomorphic to the algebra of all continuous complex-valued functions defined on a compact topological space. No corresponding result exists in the non-commutative case (see however [7]). Nevertheless in the general case it is of great inspirational value to treat elements of $A$ as "continuous complex-valued functions" defined on a topological space-like object. The latter is called a non-commutative space or pseudospace. From the formal point of view one may introduce non-commutative spaces (pseudospaces) as objects of the category dual to the category of $C^{*}$-algebras. See [17] for the details. In the present paper we do not use explicitly the pseudospace language (using instead $C^{*}$-algebra language), one should stress however that this concept stays behind many definitions and considerations presented in the following sections.

The theory of group structures on non-commutative spaces is now more than 20 years old. It was originated in [6] by Kac in an attempt to unify in one category locally compact groups and group duals and to consider generalized Pontryagin duality as a contravariant functor acting within this category.

The theory was then developed by Takesaki [11] and Schwartz and Enock [5]. In [17] it was pointed out that the right approach to the theory is the one based on the $C^{*}$-algebra language (in earlier works von Neumann algebras were used instead). In [13] Vallin developed the $C^{*}$-algebra version of the theory. Entirely different approaches are contained in recent papers of Ocneanu [10], Drinfeld [4], and Vaksman and Soibelman [14].

Despite the long history the theory seems to be still in the introductory stage. In particular the basic notions are not fixed yet. In our opinion this state was caused by the lack of interesting examples. For a long time the only examples of pseudogroups were: locally compact groups, group duals, their cartesian products and crossed products of a group dual by an automorphism group. The first example of a (not finite) compact pseudogroup of different nature was found in [18]. 
The aim of this paper is to develop the theory of compact pseudogroups in a way completely analogous to the classical theory of compact groups of matrices. In particular our main definition says that compact matrix pseudogroup is a compact pseudospace of $N \times N$ matrices closed under matrix multiplication and under taking inverses. The examples presented in Sect. 1 show that this definition gives the direct and natural generalization of the concept of the compact group of matrices. It also contains the Pontryagin duals of discrete finitely generated groups. In the world of pseudogroups $S U(2)$ and $S U(3)$ admit non-trivial deformations. At the end of Sect. 1 we show the existence of the neutral element (it is represented by ${ }^{*}$-character $e$ introduced in Proposition 1.8) and prove elementary properties linking $e$ with other basic notions.

In Sect. 2 we present the representation theory. The standard notions of intertwining operators, equivalent representations, irreducible representations, complex conjugate representations, the direct sum and the tensor product of representations are introduced and investigated. The non-commutativity of the tensor product turns out to be the property distinguishing pseudogroups from groups.

Section 3 is devoted to the concept of contragradient representation which is the main tool of the generalized Peter-Weyl theory presented in Sects. 4 and 5.

The Haar measure is the main subject of Sect. 4. We prove its existence and uniqueness and derive elementary properties. Using the Haar measure and the machinery built in Sect. 3 we prove that any smooth representation can be decomposed into a direct sum of irreducible representations. The limitation to the smooth representations is forced by the fact that our axiomatic admits cases where the Haar measure is not faithful. It becomes faithful when restricted to the subalgebra of smooth functions.

In Sect. 5 we present the Peter-Weyl theory for compact matrix pseudogroups. We prove that the matrix elements of inequivalent irreducible representations are orthogonal with respect to the two scalar product induced by the Haar measure. It turns out that the analogous formulae for matrix elements of the same irreducible representation are more complicated than in the group case. This is due to the fact that the Haar measure need not be central. It turns out that the modular properties of the Haar measure are described by a family $\left(f_{z}\right)_{z \in \mathbb{C}}$ of linear multiplicative functionals defined on the sublagebra of smooth functions and that the formulae expressing the orthonormality of matrix elements of each irreducible representation involve $f_{1}$ and $f_{-1}$.

The end of Sect. 5 is devoted to the theory of characters. We prove the basic properties and show that the character determines the representation up to equivalence.

In order to limit the volume of the paper we shift two sections devoted to the Tannaka-Krein duality and to differential calculus on compact matrix pseudogroups to separate publications $[19,20]$.

The paper contains two Appendices. In the first one we present the short proof of the Haar measure formula for $S_{\mu} U(2)$ given in [18], the second is devoted to finite matrix pseudogroups. 


\section{Definitions and Examples}

In this section we introduce the concept of compact matrix pseudogroup and present several examples. In particular we show that compact subgroups of $G L(N, \mathbb{C})$ and duals of discrete finitely generated groups are compact matrix pseudogroups. Another example can be obtained by a deformation of compact subgroups of $G L(N, \mathbb{C})$. At the end of this section we introduce the convolution product and derive simple formulae used constantly in the next sections. We start with the following basic:

Definition 1.1. Let $A$ be a $C^{*}$-algebra with unity, $u$ be a $N \times N$ matrix with entries belonging to $A: u=\left(u_{k l}\right)_{k l=1,2, \ldots, N}, u_{k l} \in A$, and $\mathscr{A}$ be the *-subalgebra of $A$ generated by the entries of $u$. We say that $(A, u)$ is a compact matrix pseudogroup if

1) $\mathscr{A}$ is dense in $A$.

2) There exists a $C^{*}$-homomorphism

$$
\Phi: A \rightarrow A \otimes A,
$$

such that

$$
\Phi\left(u_{k l}\right)=\sum_{r=1}^{N} u_{k r} \otimes u_{r l}
$$

for any $k, l=1,2, \ldots, N$.

3) There exists a linear antimultiplicative mapping

$$
\kappa: \mathscr{A} \rightarrow \mathscr{A},
$$

such that

$$
\kappa\left(\kappa\left(a^{*}\right)^{*}\right)=a
$$

for any $a \in \mathscr{A}$ and

$$
\begin{aligned}
& \sum_{r=1}^{N} \kappa\left(u_{k r}\right) u_{r l}=\delta_{k l} I, \\
& \sum_{r=1}^{N} u_{k r} \kappa\left(u_{r l}\right)=\delta_{k l} I,
\end{aligned}
$$

for any $k, l=1,2, \ldots, N . \delta_{k l}$ denotes the Kronecker symbol equal to 1 for $k=l$ and 0 otherwise, $I$ is the unity of the algebra $A$.

Due to Condition 1 the $C^{*}$-homomorphism (1.1) is uniquely determined. It will be called the comultiplication associated with $(A, u)$. Let us notice that the diagram

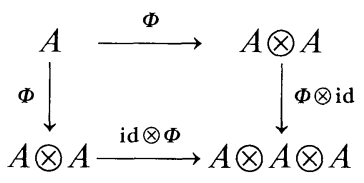

is commutative (cf. $[6,11,5,13])$. Indeed using (1.2), one easily verifies that

$$
(\mathrm{id} \otimes \Phi) \Phi\left(u_{k l}\right)=\sum_{r, s} u_{k r} \otimes u_{r s} \otimes u_{s l}=(\Phi \otimes \mathrm{id}) \Phi\left(u_{k l}\right) .
$$


Therefore $(\mathrm{id} \otimes \Phi) \Phi(a)=(\Phi \otimes \mathrm{id}) \Phi(a)$ for any $a \in \mathscr{A}$, and taking into account Condition 1 we obtain the same formula for any $a \in A$.

It follows immediately from (1.2) that

$$
\Phi(\mathscr{A}) \subset \mathscr{A} \otimes_{\text {alg }} \mathscr{A}
$$

Equations (1.5) and (1.6) show that $u$ is an invertible element of $M_{N}(A)$ and that

$$
\kappa\left(u_{k l}\right)=u^{-1}{ }_{k l},
$$

where $u^{-1}{ }_{k l}$ denote the matrix elements of the inverse of $u$. For any $a \in \mathscr{A}$ we set

$$
a^{\#}=\kappa\left(a^{*}\right) \text {. }
$$

By virtue of Condition 3, \# is an antilinear involution acting on $\mathscr{A}$ and $\kappa(a)^{\#}=a^{*}$ for any $a \in \mathscr{A}$. Applying \# to the both sides of (1.5) and (1.6) we obtain

$$
\begin{aligned}
& \sum_{r} u_{k r}^{*} \kappa\left(u_{r l}{ }^{*}\right)=\delta_{k l} I, \\
& \sum_{r} \kappa\left(u_{k r}{ }^{*}\right) u_{r l}{ }^{*}=\delta_{k l} I,
\end{aligned}
$$

for any $k, l=1,2, \ldots, N$. Let $\bar{u}$ denote the $N \times N$ matrix with matrix elements being the hermitian conjugate of elements of $u: \bar{u}=\left(\bar{u}_{k l}\right)_{k l=1,2, \ldots, N}$ and $\bar{u}_{k l}=u_{k l}{ }^{*}$. Equations (1.11) and (1.12) show that $\bar{u}$ is an invertible element of $M_{N}(A)$ and that

$$
\kappa\left(u_{k l}^{*}\right)=\bar{u}^{-1}{ }_{k l},
$$

where $\bar{u}^{-1}{ }_{k l}(k, l=1,2, \ldots, N)$ denote the matrix elements of the inverse of $\bar{u}$.

Remembering that the algebra $\mathscr{A}$ is generated by $u_{k l}$ and $u_{k l}{ }^{*}(k, l=1,2, \ldots, N)$ and taking into account (1.9) and (1.13) we see that the mapping (1.3) is uniquely determined. It will be called the coinverse associated with $(A, u)$.

Let $\sigma_{A}$ denote the flip automorphism of $A \otimes A$ :

$$
\sigma_{A}(a \otimes b)=b \otimes a
$$

for any $a, b \in A$ and $A \otimes_{\text {sym }} A=\left\{x \in A \otimes A: \sigma_{A}(x)=x\right\}$. We say that a pseudogroup $(A, u)$ is abelian if $\Phi(A) \subset A \otimes_{\text {sym }} A$.

Let $\left(A,\left(u_{k l}\right)_{k l=1,2, \ldots, N}\right)$ and $\left(A^{\prime},\left(u_{k l}^{\prime}\right)_{k l=1,2, \ldots, N^{\prime}}\right)$ be compact matrix pseudogroups. We say that they are identical if $N^{\prime}=N$, and if there exists a $C^{*}$-isomorphism $s$ of $A$ onto $A^{\prime}$ such that

$$
s\left(u_{k l}\right)=u_{k l}^{\prime}
$$

for $k, l=1,2, \ldots, N$. In that case $s$ is uniquely determined, it maps the *-subalgebra $\mathscr{A}$ of $A$ generated by matrix elements of $u$ onto the corresponding *-subalgebra $\mathscr{A}^{\prime}$ related to $\left(A^{\prime}, u^{\prime}\right)$. Moreover denoting by $\Phi, \kappa$ and $\Phi^{\prime}, \kappa^{\prime}$ the comultiplications and the coinverses associated with $(A, u)$ and $\left(A^{\prime}, u^{\prime}\right)$ respectively we have the following commutative diagrams

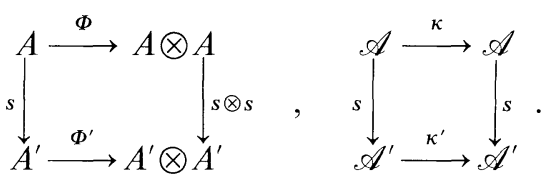


The latter follows immediately from the uniqueness of the comultiplication and the coinverse maps. From the abstract point of view the identical pseudogroups are undistinguishable.

Let $\left(A,\left(u_{k l}\right)_{k l=1,2, \ldots, N}\right)$ be a compact matrix pseudogroup, $t=\left(t_{r s}\right)_{r s=1,2, \ldots, N}$ be an invertible matrix with complex entries and

$$
v_{k l}=\sum_{r, s} t^{-1}{ }_{k r} u_{r s} t_{s l} .
$$

One can easily check that $\left(A,\left(v_{k l}\right)_{k l=1,2, \ldots, N}\right)$ is a compact matrix pseudogroup. The dense *-subalgebra $\mathscr{A}$, the comultiplication $\Phi$ and the coinverse $\kappa$ are the same for $(A, u)$ and $(A, v)$. We say that $(A, v)$ is obtained from $(A, u)$ by the similarity transformation. Two compact matrix pseudogroups $(A, u)$ and $\left(A^{\prime}, u^{\prime}\right)$ are said to be similar if the pseudogroup obtained from $(A, u)$ by a similarity transformation and $\left(A^{\prime}, u^{\prime}\right)$ are identical.

\section{Examples}

I. Pseudogroups with Commutative $C^{*}$-Algebras. Let $G$ be a compact group of $N \times N$ matrices with complex entries: $G \subset M_{N}(\mathbb{C})$. We denote by $C(G)$ the commutative $C^{*}$-algebra of all continuous functions on $G$. For any $g \in G$ and any $k, l=1,2, \ldots, N$, we denote by $w_{k l}(g)$ the matrix element of $g$ standing in the $k^{\text {th }}$ row and the $l^{\text {th }}$ column:

$$
g=\left(w_{k l}(g)\right)_{k l=1,2, \ldots, N} .
$$

Clearly $w_{k l}(g)$ depends continuously on $g$, i.e. $w_{k l}$ are continuous functions defined on $G: w_{k l} \in C(G)$. Let $w_{G}=\left(w_{k l}\right)_{k l=1,2, \ldots, N}$. Then we have

Theorem 1.1. $\left(C(G), w_{G}\right)$ is a compact matrix pseudogroup.

Proof. Condition 1 follows immediately from the Stone-Weierstrass theorem [9]. To prove Condition 2 we identify $C(G) \otimes C(G)$ with $C(G \times G)$. Then

$$
(a \otimes b)\left(g, g^{\prime}\right)=a(g) b\left(g^{\prime}\right)
$$

for any $a, b \in C(G)$ and $g, g^{\prime} \in G$.

According to the wellknown rules of matrix calculus the matrix element of the product of two matrices $g, g^{\prime} \in G$ standing in $k^{\text {th }}$ row and $l^{\text {th }}$ column equals the product of the $k^{\text {th }}$ row of, $g$ by $l^{\text {th }}$ column of $g^{\prime}$. It means that

$$
w_{k l}\left(g g^{\prime}\right)=\sum_{r} w_{k r}(g) w_{r l}\left(g^{\prime}\right)
$$

For any $a \in C(G)$ and $g, g^{\prime} \in G$, we set

$$
(\Phi(a))\left(g, g^{\prime}\right)=a\left(g g^{\prime}\right) .
$$

Clearly $\Phi(a) \in C(G \times G)$ and $\Phi: C(G) \rightarrow C(G) \otimes C(G)$ is a homomorphism in the category of $C^{*}$-algebras. Moreover taking into account (1.17) and (1.16), we see that

$$
\Phi\left(w_{k l}\right)=\sum_{r} w_{k r} \otimes w_{r l}
$$

and Condition 2 of Definition 1.1 is fulfilled. 
For any $a \in C(G)$ and $g \in G$, we set

$$
(\kappa(a))(g)=a\left(g^{-1}\right) .
$$

Clearly $\kappa(a) \in C(G)$ and $\kappa: C(G) \rightarrow C(G)$ is a linear multiplicative (notice that in the considered commutative case multiplicativity and antimultiplicativity are equivalent) and involutive (i.e. $\kappa^{2}=\mathrm{id}$ ) mapping. Moreover

$$
\kappa\left(a^{*}\right)=\kappa(s)^{*} .
$$

Therefore $\kappa\left(\kappa\left(a^{*}\right)^{*}\right)=\kappa\left(\kappa(a)^{* *}\right)=\kappa(\kappa(a))=a$ for any $a \in C(G)$.

Inserting in (1.17) $g^{-1}$ instead of $g^{\prime}$ and using (1.15) we obtain (1.6). Similarly replacing in (1.17) $g$ and $g^{\prime}$ by $g^{-1}$ and $g$ respectively and taking into account $(1.15)$ we get (1.5).

To end the proof we have to show that $\kappa(\mathscr{A}) \subset \mathscr{A}$, where $\mathscr{A}$ is the $*_{\text {-subalgebra }}$ of $C(G)$ generated by functions $w_{k l}(k, l=1,2, \ldots, N)$. It is known [1] that any representation of a compact group is equivalent to a unitary one. Therefore there exists a strictly positive matrix $m \in M_{N}(\mathbb{C})$ such that $g^{*} m g=m$ for any $g \in G$. Therefore $g^{-1}=m^{-1} g^{*} m$ for any $g \in G$. Using this relation one easily verifies that

$$
\kappa\left(w_{k l}\right)=\sum_{r, s} m_{k r}^{-1} m_{s l} w_{s r}^{*},
$$

where $m^{-1}{ }_{k r}$ and $m_{s l}$ are matrix elements of $m^{-1}$ and $m$ respectively. This equation shows that $\kappa\left(w_{k l}\right) \in \mathscr{A}$ for all $k, l=1,2, \ldots, N$, and using the multiplicativity of $\kappa$ and (1.20), we get $\kappa(a) \in \mathscr{A}$ for any $a \in \mathscr{A}$. Q.E.D.

Let us notice that $c \in C(G) \otimes_{\text {sym }} C(G)$ if and only if $c\left(g, g^{\prime}\right)=c\left(g^{\prime}, g\right)$ for any $g, g^{\prime} \in G$. Taking into account (1.18) we get

Proposition 1.2. The pseudogroup $\left(C(G), w_{G}\right)$ is abelian if and only if $g g^{\prime}=g^{\prime} g$ for any $g, g^{\prime} \in G$.

For any $g \in G$ and $a \in C(G)$ we set $\chi_{g}(a)=a(g)$. It is well known that $\chi_{g}$ is a character of $C(G)$ and that $\left\{\chi_{g}: g \in G\right\}$ coincides with the set of all characters of $C(G)$. Let us notice that $\left(\chi_{g}\left(w_{k l}\right)\right)_{k l=1,2, \ldots, N}=\left(w_{k l}(g)\right)_{k l=1,2, \ldots, N}=g$ for any $g \in G$. Therefore

$$
G=\left\{\left(\chi\left(w_{k l}\right)\right)_{k l=1,2, \ldots, N}: \chi \text { is a character of } C(G)\right\} .
$$

Clearly the right-hand side of the above equation remains unchanged if we replace $\left(C(G), w_{G}\right)$ by a pseudogroup identical with $\left(C(G), w_{G}\right)$. Therefore we have

Proposition 1.3. Let $G$ and $G^{\prime}$ be compact group of matrices. The pseudogroups $\left(C(G), w_{G}\right)$ and $\left(C\left(G^{\prime}\right), w_{G}\right)$ are identical if and only if $G=G^{\prime}$.

One can also prove the following

Proposition 1.4. Let $G$ and $G^{\prime}$ be a compact group of $N \times N$ matrices. The pseudogroups $\left(C(G), w_{G}\right)$ and $\left(C\left(G^{\prime}\right), w_{G}\right)$ are similar if and only if there exists an invertible matrix $t \in M_{N}(\mathbb{C})$ such that $G^{\prime}=\left\{t^{-1} g t: g \in G\right\}$.

It turns out that the construction described above produces all compact matrix pseudogroups $(A, u)$ with commutative $A$. More precisely we have 
Theorem 1.5. Let $\left(A,\left(u_{k l}\right)_{k l=1,2, \ldots, N}\right)$ be a compact matrix pseudogroup. Assume that $A$ is commutative. Then

1) The set

$$
G=\left\{\left(\chi\left(u_{k l}\right)\right)_{k l=1,2, \ldots, N}: \chi \text { is a character of } A\right\}
$$

is a compact group of matrices.

2) The pseudogroups $(A, u)$ and $\left(C(G), w_{G}\right)$ (where $G$ is given by (1.21)) are identical.

Proof. Let $A$ be the set of all characters of $A$ and $j$ be the mapping defined on $A$ with values in $M_{N}(\mathbb{C})$ such that

$$
j(\chi)=\left(\chi\left(u_{k l}\right)\right)_{k l=1,2, \ldots, N}
$$

for any $\chi \in \Lambda$. Clearly [cf. (1.21)] $G$ is the $j$-image of $\Lambda$.

It is obvious that $j$ is continuous with respect to the pointwise convergence topology on $A$. On the other hand (cf. the Gelfand-Naimark theory of commutative $C^{*}$-algebras [3]) $\Lambda$ endowed with this topology is a compact space. It shows that $G$ is a compact subset of $M_{N}(\mathbb{C})$.

Let $\chi \in A$. Applying $\chi$ to the both sides of (1.5) and (1.6) we see that the matrix $\left(\chi \circ \kappa\left(u_{k l}\right)\right)_{k l=1,2, \ldots, N}$ coincides with the inverse of $j(\chi)$. It shows that all matrices belonging to $G$ are invertible.

Let $\chi$ and $\chi^{\prime}$ be characters of $A$ and $\chi^{\prime \prime}=\left(\chi \otimes \chi^{\prime}\right) \Phi$. Then $\chi^{\prime \prime}$ is a character of $A$ and using (1.2) we see that $j\left(\chi^{\prime \prime}\right)=j(\chi) j\left(\chi^{\prime}\right)$. It shows that the product of two matrices belonging to $G$ belongs to $G$.

Let $g \in G$. Then $g^{n} \in G$ for any natural $n$. Since $G$ is compact, one can find a sequence of natural numbers $(n(k))_{k \in \mathbb{N}}$ such that $n(k+1)>n(k)+1(k=1,2, \ldots)$ and $g^{n(k)} \rightarrow g_{\infty}$ when $k \rightarrow \infty$. Then $m(k)=n(k+1)-(1+n(k))>1, g^{m(k)}$ belongs to $G$ and

$$
\lim _{k \rightarrow \infty} g^{m(k)}=g_{\infty}\left(g g_{\infty}\right)^{-1}=g^{-1} .
$$

It shows that $g^{-1} \in G$. This way Statement 1 is proved.

Let $a \in C(G)$. Then $a \circ j$ is a continuous function on $\Lambda$ and according to the Gelfand-Naimark theory there exists a unique element $s a \in A$ such that

$$
\chi(s a)=a(j(\chi))
$$

for any $\chi \in \Lambda$. Clearly the mapping

$$
C(G) \ni a \rightarrow s a \in A
$$

is a $C^{*}$-homomorphism. If $s a=0$ then $a(j(\chi))=0$ for any $\chi \in \Lambda$. Therefore $a(g)=0$ for any $g \in G$ and $a=0$. It shows that (1.24) is an embedding.

Comparing (1.15) and (1.22) we see that $w_{k l}(j(\chi))=\chi\left(u_{k l}\right)$ for any $\chi \in \Lambda$. Therefore [cf. (1.23)]

$$
S w_{k l}=u_{k l}
$$

for $k, l=1,2, \ldots, N$. Remembering (Condition 1 of Definition 1.1) that algebraic combinations of elements $u_{k l}$ form a dense subset of $A$, we see that (1.24) is onto. Therefore $s$ is a $C^{*}$-isomorphism and (1.25) shows that the pseudogroups $\left(C(G), w_{G}\right)$ and $(A, u)$ are identical. Q.E.D. 
II. Abelian Pseudogroups. Let $\Gamma$ be a (discrete) group generated by a finite subset $\left\{\gamma_{1}, \gamma_{2}, \ldots, \gamma_{N}\right\} \subset \Gamma$ and $U$ be a unitary faithful representation of $\Gamma$ acting on a Hilbert space $H$. We shall assume that:

$$
\left(\begin{array}{c}
U \otimes U \text { is contained } \\
\text { in a multiple of } U
\end{array}\right) .
$$

This assumption is obviously fulfilled if $U=U_{\text {univ }}$, where $U_{\text {univ }}$ is the direct sum of all cyclic representations. It is also satisfied for $U=U_{\text {reg, }}$, where $U_{\text {reg }}$ is the right regular representation of $\Gamma$ (cf. [12]).

Let $C^{*}(U)$ be the $C^{*}$-algebra of operators acting on $H$ generated by $\{U(\gamma): \gamma \in \Gamma\}$ and $u$ be the $N \times N$ matrix having $U\left(\gamma_{1}\right), U\left(\gamma_{2}\right), \ldots, U\left(\gamma_{N}\right)$ on the diagonal and zeroes in all other places. Then we have

Theorem 1.6. $\left(C^{*}(U), u\right)$ is an abelian compact matrix pseudogroup.

Proof. Let $\mathscr{A}$ be the *-subalgebra of $C^{*}(U)$ generated by matrix elements of $u$, i.e. by $U\left(\gamma_{1}\right), U\left(\gamma_{2}\right), \ldots, U\left(\gamma_{N}\right)$. Remembering that $U$ is unitary representation and that $\left\{\gamma_{1}, \gamma_{2}, \ldots, \gamma_{N}\right\}$ generate $\Gamma$, one immediately sees that $\{U(\gamma): \gamma \in \Gamma\} \subset \mathscr{A}$. Therefore $\mathscr{A}$ is dense in $C^{*}(U)$.

Assumption (1.26) means that there exists a Hilbert space $K$ and an isometry $W: H \otimes H \rightarrow K \otimes H$ such that

$$
U(\gamma) \otimes U(\gamma)=W^{*}\left(I_{B(K)} \otimes U(\gamma)\right) W
$$

for any $\gamma \in \Gamma$. For any $a \in C^{*}(U)$, we set

$$
\Phi(a)=W^{*}\left(I_{B(K)} \otimes a\right) W .
$$

By virtue of (1.27),

$$
\Phi(\mathrm{U}(\gamma))=\mathrm{U}(\gamma) \otimes U(\gamma) .
$$

Clearly $U(\gamma) \otimes U(\gamma) \in C^{*}(U) \otimes_{\text {sym }} C^{*}(U)$. Therefore $\Phi$ is a $C^{*}$-homomorphism acting from $C^{*}(U)$ into $C^{*}(U) \otimes_{\text {sym }} C^{*}(U)$. Inserting in $(1.29) \gamma=\gamma_{1}, \gamma_{2}, \ldots, \gamma_{N}$, we see that (1.2) is satisfied.

Any element $a \in \mathscr{A}$ is of the form

$$
a=\sum_{\gamma \in \Gamma_{0}} a_{\gamma} U(\gamma)
$$

where $a_{\gamma}$ are complex coefficients and $\Gamma_{0}$ is a finite subset of $\Gamma$.

Assume that $a=0$. Then applying $(k-1)$-times the mapping $\Phi$ to the both sides of (1.30) we obtain

$$
\sum_{\nu \in \Gamma_{0}} a_{\gamma} \underbrace{U(\gamma) \otimes U(\gamma) \otimes \ldots \otimes U(\gamma)}_{k \text {-factors }}=0
$$

and

$$
\sum_{\gamma \in \Gamma_{0}} a_{\gamma}[\lambda(U(\gamma))]^{k}=0
$$

for any linear functional $\lambda$ defined on $C^{*}(U)$ and any number $k$. In what follows we take $k=1,2, \ldots, K$, where $K=$ number of elements of $\Gamma_{0}$. Since all elements $U(\gamma)$. 
$\left(\gamma \in \Gamma_{0}\right)$ are different (representation $U$ is faithful) and nonvanishing, one can find $\lambda$ such that all $\lambda(U(\gamma))\left(\gamma \in \Gamma_{0}\right)$ are nonvanishing and different. We know that in this case the Vandermonde determinant nonvanishes and (1.31) implies that all $a_{\gamma}=0$. It shows that coefficients $a_{\gamma}$ in (1.30) are uniquely determined by $a$.

Using this result one can easily show that there exists a linear map $\kappa: \mathscr{A} \rightarrow \mathscr{A}$ such that

$$
\kappa(U(\gamma))=U\left(\gamma^{-1}\right)
$$

for any $\gamma$. Clearly $\kappa$ is antimultiplicative and $\kappa\left(\kappa\left(a^{*}\right)^{*}\right)=a$ for any $a \in \mathscr{A}$. Inserting in (1.32) $\gamma=\gamma_{1}, \gamma_{2}, \ldots, \gamma_{N}$, we see that (1.5) and (1.6) are satisfied. Q.E.D.

If $U=U_{\text {univ }}\left(=U_{\text {reg }}\right.$ respectively) then the pseudogroup $\left(C^{*}(U), u\right)$ is called universal (regular respectively) dual of $\Gamma$. If $\Gamma$ is amenable then the two duals are identical. If $\Gamma$ is abelian then $C^{*}\left(U_{\text {reg }}\right)$ is commutative. In this case (cf. Theorem 1.6 and Proposition 1.2) the dual of $\Gamma$ is identical with $\left(C(G), w_{G}\right)$, where $G$ is an abelian compact group of matrices. Then $G$ is Pontryagin dual of $\Gamma$.

It turns out that the construction described above produces all (up to a similarity) abelian compact matrix pseudogroups. In Sect. 4 we prove the following

Theorem 1.7. Let $\left(A,\left(u_{k l}\right)_{k l=1,2, \ldots, N}\right)$ be an abelian compact matrix pseudogroup. Then there exists a (discrete) group $\Gamma$ generated by $N$ elements $\gamma_{1}, \gamma_{2}, \ldots, \gamma_{N}$ and a faithful representation $U$ of $\Gamma$ satisfying condition (1.26) such that the pseudogroups $\left(A,\left(u_{k l}\right)_{k l=1,2, \ldots, N}\right)$ and $\left(C^{*}(U),\left(U\left(\gamma_{k}\right) \delta_{k l}\right)_{k l=1,2, \ldots, N}\right)$ are similar.

Proof. See the text following the proof of Theorem 4.5.

The group $\Gamma$ is called the dual of $(A, u)$. If $A$ is commutative then $(A, u)$ is identical with $\left(C(G), w_{G}\right)$, where $G$ is an abelian compact group of matrices. In this case $\Gamma$ is the Pontryagin dual of $G$.

III. Twisted $S U(N)$ Groups. A. Let $\mu$ be a nonzero real number in the interval $[-1,1]$ and $A$ be the $C^{*}$-algebra generated by two elements $\alpha$ and $\gamma$ satisfying the following relations:

$$
\begin{gathered}
\alpha^{*} \alpha+\gamma^{*} \gamma=I, \quad \alpha \alpha^{*}+\mu^{2} \gamma \gamma^{*}=I, \\
\gamma \gamma^{*}=\gamma^{*} \gamma, \quad \mu \gamma \alpha=\alpha \gamma, \quad \mu \gamma^{*} \alpha=\alpha \gamma^{*} .
\end{gathered}
$$

We consider $2 \times 2$ matrix

$$
u=\left(\begin{array}{cc}
\alpha, & -\mu v^{*} \\
v, & \alpha^{*}
\end{array}\right)
$$

Then $(A, u)$ is a compact matrix pseudogroup (see [18] for details and proofs). If $\mu=1$ then $A$ is commutative and $(A, u)$ is identical with $\left(C(G), w_{G}\right)$, where $G=S U(2)$. In the general case $(A, u)$ is called the twisted $S U(2)$ group and denoted by $S_{\mu} U(2)$.

B. Let $\mu \in] 0,1]$. We introduce a 27 element array $\left(E_{k l m}\right)$, where $k, l, m=1,2,3$ :

$$
\begin{aligned}
E_{231}= & E_{312}=\mu^{2}, \quad E_{213}=E_{132}=-\mu, \\
E_{123} & =1, \quad E_{321}=-\mu^{3} .
\end{aligned}
$$


All other elements $E_{k l m}$ vanish.

Let $A$ be the $C^{*}$-algebra generated by 9 elements $u_{k l}(k, l=1,2,3)$ satisfying the following relations

$$
\begin{gathered}
\sum_{r} u_{r k}^{*} u_{r l}=\sigma_{k l} I, \\
\sum_{r} u_{k r} u_{l r}^{*}=\delta_{k l} I, \\
\sum_{p q r} u_{k p} u_{l q} u_{m r} E_{p q r}=E_{k l m} I,
\end{gathered}
$$

for any $k, l, m=1,2,3$.

Then $\left(A,\left(u_{k l}\right)_{k l=1,2,3}\right)$ is a compact matrix pseudogroup. It will be denoted by $S_{\mu} U(3)$. If $\mu=1$, then the algebra $A$ is commutative and $S_{\mu} U(3)$ is identical with $\left(C(S U(3)), w_{S U(3)}\right)$. See [2] for details and proofs.

C. The general definition of twisted $S U(N)$ group will be given in [19].

We end this section with elementary consequences of Conditions 1-3 of Definition 1.1. In the following propositions $(A, u)$ is a compact matrix pseudogroup, $\mathscr{A}$ is the dense ${ }^{*}$-subalgebra of $A$ generated by matrix elements of $u, \Phi$, and $\kappa$ denote comultiplication and coinverse associated with $(A, u)$.

We shall also use the linear map

$$
m: \mathscr{A} \otimes_{\text {alg }} \mathscr{A} \rightarrow \mathscr{A},
$$

such that $m(a \otimes b)=a b$ for any $a, b \in \mathscr{A}$.

Proposition 1.8. There exists one and only one *-character,

$$
e: \mathscr{A} \rightarrow \mathbb{C}
$$

such that for all $k, l=1,2, \ldots, N$ we have

$$
e\left(u_{k l}\right)=\delta_{k l} \text {. }
$$

Moreover for any $a \in \mathscr{A}$ we have

$$
\begin{aligned}
& m(\kappa \otimes \mathrm{id}) \Phi(a)=e(a) I, \\
& m(\mathrm{id} \otimes \kappa) \Phi(a)=e(a) I .
\end{aligned}
$$

Remark. Due to (1.8) the left-hand sides of (1.38) and (1.39) are well defined.

Remark. Often $e$ is norm continuous and then it can be extended to the whole $A$. This is the case for $\left(C(G), w_{G}\right)$ [then $e(a)$ is the value of $a$ at the neutrale element of $G],\left(C^{*}\left(U_{\text {univ }}\right), u\right), S_{\mu} U(2)$, and $S_{\mu} U(3)$. If $\Gamma$ is not amenable then for the $\left(C^{*}\left(U_{\text {reg }}\right), u\right)$ case $e$ is not continuous.

Proof. Since $\mathscr{A}$ is a *algebra generated by $u_{k l}(k, l=1,2, \ldots, N)$, any *-character on $\mathscr{A}$ is determined by the values it assumes on $u_{k l}$. This remark proves the uniqueness of $e$.

Let

$$
\mathscr{A}_{0}=\left\{\begin{array}{c}
\text { There exists a complex number } e(a) \\
a \in \mathscr{A}: \text { such that } m(\kappa \otimes \mathrm{id}) \Phi(a)=e(a) I \\
\text { and } m(\mathrm{id} \otimes \kappa) \Phi(a)=e(a) I
\end{array}\right\}
$$


Clearly $\mathscr{A}_{0}$ is a linear subset of $\mathscr{A}$ and $e$ is a linear functional defined on $\mathscr{A}_{0}$. Taking into account (1.2), (1.5), and (1.6), we have

$$
\begin{aligned}
& m(\kappa \otimes \mathrm{id}) \Phi\left(u_{k l}\right)=\sum_{r} \kappa\left(u_{k r}\right) u_{r l}=\delta_{k l} I, \\
& m(\mathrm{id} \otimes \kappa) \Phi\left(u_{k l}\right)=\sum_{r} u_{k r} \kappa\left(u_{r l}\right)=\delta_{k l} I .
\end{aligned}
$$

Therefore

$$
\left(\begin{array}{c}
u_{k l} \in \mathscr{A}_{0} \text { and } \\
e\left(u_{k l}\right)=\delta_{k l} \text { for } \\
k, l=1,2, \ldots, N
\end{array}\right)
$$

We shall show that

$$
\left(\begin{array}{c}
\mathscr{A}_{0} \text { is } * \text {-invariant } \\
\text { and } e\left(a^{*}\right)=\overline{e(a)} \\
\text { for any } a \in \mathscr{A}_{0}
\end{array}\right) .
$$

Let $a \in \mathscr{A}$ and

$$
\Phi(a)=\sum_{r} a_{r}^{\prime} \otimes a_{r}^{\prime \prime}
$$

where $a_{r}^{\prime}, a_{r}^{\prime \prime} \in \mathscr{A}, r=1,2, \ldots, R$. Assume that $a \in \mathscr{A}_{0}$. Then

$$
\begin{aligned}
& \sum_{r} \kappa\left(a_{r}^{\prime}\right) a_{r}^{\prime \prime}=m(\kappa \otimes \mathrm{id}) \Phi(a)=e(a) I, \\
& \sum_{r} a_{r}^{\prime} \kappa\left(a_{r}^{\prime \prime}\right)=m(\mathrm{id} \otimes \kappa) \Phi(a)=e(a) I .
\end{aligned}
$$

Applying to both sides of these relations the antilinear multiplicative involution \# introduced by (1.10), we obtain

$$
\begin{aligned}
& \sum_{r} \mathrm{a}_{r}^{\prime *} \kappa\left(\mathrm{a}_{r}^{\prime \prime *}\right)=\overline{e(a)} I, \\
& \sum_{r} \kappa\left(a_{r}^{\prime *}\right) a_{r}^{\prime \prime *}=\overline{e(a)} I .
\end{aligned}
$$

Therefore

$$
\begin{aligned}
m(\kappa \otimes \mathrm{id}) \Phi\left(a^{*}\right) & =m(\kappa \otimes \mathrm{id}) \sum_{r} a_{r}^{\prime *} \otimes a_{r}^{\prime *} \\
& \left.=\sum_{r} \kappa\left(a_{r}^{\prime *}\right) a_{r}^{\prime *}=\overline{e(a}\right) I \\
m(\mathrm{id} \otimes \kappa) \Phi\left(a^{*}\right) & =m(\mathrm{id} \otimes \kappa) \sum_{r} a_{r}^{\prime *} \otimes a_{r}^{\prime \prime *} \\
& =\sum_{r} a_{r}^{\prime *} \kappa\left(a_{r}^{\prime \prime *}\right)=\overline{e(a)} I .
\end{aligned}
$$

These relations show that $a^{*} \in \mathscr{A}_{0}$ and $e\left(a^{*}\right)=e(a)$. Statement (1.41) is proved.

We shall show that

$$
\left(\begin{array}{c}
\mathscr{A}_{0} \text { is a subalgebra of } \mathscr{A} \\
\text { and the functional } e \\
\text { is multiplicative }
\end{array}\right) .
$$


Let $a, b \in \mathscr{A}$ and

$$
\Phi(a)=\sum_{r} a_{r}^{\prime} \otimes a_{r}^{\prime \prime}, \quad \Phi(b)=\sum_{s} b_{s}^{\prime} \otimes b_{s}^{\prime \prime},
$$

where $a_{r}^{\prime}, a_{r}^{\prime \prime}, b_{s}^{\prime}, b_{s}^{\prime \prime} \in \mathscr{A}, r=1,2, \ldots, R, s=1,2, \ldots, S$. Assume that $a, b \in \mathscr{A}_{0}$. Then

$$
\begin{aligned}
& \sum_{r} \kappa\left(a_{r}^{\prime}\right) a_{r}^{\prime \prime}=m(\kappa \otimes \mathrm{id}) \Phi(a)=e(a) I, \\
& \sum_{s} \kappa\left(b_{s}^{\prime}\right) b_{s}^{\prime \prime}=m(\kappa \otimes \mathrm{id}) \Phi(b)=e(b) I, \\
& \sum_{r} a_{r}^{\prime} \kappa\left(a_{r}^{\prime \prime}\right)=m(\mathrm{id} \otimes \kappa) \Phi(a)=e(a) I, \\
& \sum_{s} b_{s}^{\prime} \kappa\left(b_{s}^{\prime \prime}\right)=m(\mathrm{id} \otimes \kappa) \Phi(b)=e(b) I .
\end{aligned}
$$

We compute

$$
\Phi(a b)=\sum_{r, s} a_{r}^{\prime} b_{s}^{\prime} \otimes a_{r}^{\prime \prime} b_{s}^{\prime \prime} .
$$

Using the antimultiplicativity of $\kappa,(1.43)$ and (1.44) we get

$$
\begin{aligned}
m(\kappa \otimes \mathrm{id}) \Phi(a b) & =\sum_{r, s} \kappa\left(a_{r}^{\prime} b_{s}^{\prime}\right) a_{r}^{\prime \prime} b_{s}^{\prime \prime}=\sum_{r, s} \kappa\left(b_{s}^{\prime}\right) \kappa\left(a_{r}^{\prime}\right) a_{r}^{\prime \prime} b_{s}^{\prime \prime} \\
& =e(a) \sum_{s} \kappa\left(b_{s}^{\prime}\right) b_{s}^{\prime \prime}=e(a) e(b) I .
\end{aligned}
$$

Similarly using the antimultiplicativity of $\kappa,(1.45)$ and (1.46) we obtain

$$
m(\mathrm{id} \otimes \kappa) \Phi(a b)=e(a) e(b) I .
$$

The last two relations show that $a b \in \mathscr{A}_{0}$ and that $e(a b)=e(a) e(b)$. Statement (1.42) is proved.

According to (1.41), (1.42), and (1.40), $\mathscr{A}_{0}$ is a ${ }^{*}$-subalgebra of $\mathscr{A}$ containing all matrix elements of $u$. Therefore $\mathscr{A}_{0}=\mathscr{A}$. Moreover $e$ is a $*$-character on $\mathscr{A}$, $e\left(u_{k l}\right)=\delta_{k l}(k, l=1,2, \ldots, N)$, and relations (1.38) and (1.39) are satisfied for all $a \in \mathscr{A}$. Q.E.D.

Proposition 1.9. For any $a \in \mathscr{A}$,

$$
\Phi(\kappa(a))=\sigma_{A}(\kappa \otimes \kappa) \Phi(a),
$$

Proof. Let us notice that $\Phi \circ \kappa$ and $\sigma_{A} \circ(\kappa \otimes \kappa) \circ \Phi$ are linear antimultiplicative mappings defined on $\mathscr{A}$. Therefore it is sufficient to prove (1.47) for $a=u_{k l}$ and $a=u_{k l}^{*}(k, l=1,2, \ldots, N)$.

Applying $\Phi$ to both sides of (1.5) and using (1.2), we obtain

$$
\sum_{r, s} \Phi\left(\kappa\left(u_{k r}\right)\right)\left(u_{r s} \otimes u_{s l}\right)=\delta_{k l} I \otimes I .
$$

Multiplying both sides from the right by $\sum_{n} \kappa\left(u_{n m}\right) \otimes \kappa\left(u_{l n}\right)$, summing over $l$ and using twice (1.6), we get

$$
\begin{aligned}
\Phi\left(\kappa\left(u_{k m}\right)\right) & =\sum_{n} \kappa\left(u_{n m}\right) \otimes \kappa\left(u_{k n}\right) \\
& =\sigma_{A}(\kappa \otimes \kappa) \sum_{n} u_{k n} \otimes u_{n m}=\sigma_{A}(\kappa \otimes \kappa) \Phi\left(u_{k m}\right) .
\end{aligned}
$$


Similarly applying $\Phi$ to both sides of (1.12) and using (1.2), we obtain

$$
\sum_{r, s} \Phi\left(\kappa\left(u_{k r}^{*}\right)\right)\left(u_{r s}^{*} \otimes u_{s l}^{*}\right)=\delta_{k l} I \otimes I
$$

Multiplying both sides from the right by $\sum_{n} \kappa\left(u_{n m}{ }^{*}\right) \otimes \kappa\left(u_{l n}{ }^{*}\right)$, summing over $l$ and using twice (1.11), we get

$$
\begin{aligned}
\Phi\left(\kappa\left(u_{k m}{ }^{*}\right)\right) & =\sum_{n} \kappa\left(u_{n m}{ }^{*}\right) \otimes \kappa\left(u_{k n}{ }^{*}\right) \\
& =\sigma_{A}(\kappa \otimes \kappa) \sum_{n} u_{k n}{ }^{*} \otimes u_{n m}{ }^{*}=\sigma_{A}(\kappa \otimes \kappa) \Phi\left(u_{k m}{ }^{*}\right) .
\end{aligned}
$$

Now we have to introduce convolution products. Let $\xi$ and $\xi^{\prime}$ be continuous linear functionals on $A$ and $a \in A$. Then we set:

$$
\begin{aligned}
& \xi * a=(\mathrm{id} \otimes \xi) \Phi(a), \\
& a * \xi^{\prime}=\left(\xi^{\prime} \otimes \mathrm{id}\right) \Phi(a), \\
& \xi^{\prime} * \xi=\left(\xi^{\prime} \otimes \xi\right) \circ \Phi .
\end{aligned}
$$

Clearly $\xi * a$ and $a * \xi^{\prime}$ belong to $A ; \xi^{\prime} * \xi$ is a continuous linear functional on $A$. The commutativity of the diagram (1.7) implies the associativity of the convolution product. Moreover we have

$$
\left(\xi^{\prime} * \xi\right)(a)=\xi\left(a * \xi^{\prime}\right)=\xi^{\prime}(\xi * a) .
$$

Due to (1.8) the right-hand sides of (1.48)-(1.50) are meaningful for any linear functionals $\xi, \xi^{\prime}$ defined on $\mathscr{A}$ and any $a \in \mathscr{A}$. In this case $\xi * a, a * \xi^{\prime} \in \mathscr{A}$, and $\xi^{\prime} * \xi$ is a linear functional defined on $\mathscr{A}$.

The convolution product is commutative if and only if the pseudogroup is abelian.

Let $e$ be the functional on $\mathscr{A}$ introduced in Proposition 1.8. Then

$$
e * a=a * e=a
$$

for any $a \in \mathscr{A}$. Indeed mappings,

$$
\begin{aligned}
& a \rightarrow e * a=(\mathrm{id} \otimes e) \Phi(a), \\
& a \rightarrow a * e=(e \otimes \mathrm{id}) \Phi(a),
\end{aligned}
$$

are linear, multiplicative and commute with the hermitian conjugation. Therefore it is sufficient to check (1.52) for $a=u_{k l}(k, l=1,2, \ldots, N)$. Using (1.2) and (1.37), we get

$$
\begin{aligned}
& e * u_{k l}=(\mathrm{id} \otimes e) \sum_{r} u_{k r} \otimes u_{r l}=\sum_{r} u_{k r} \delta_{r l}=u_{k l}, \\
& u_{k l} * e=(e \otimes \mathrm{id}) \sum_{r} u_{k r} \otimes u_{r l}=\sum_{r} \delta_{k r} u_{r l}=u_{k l} .
\end{aligned}
$$

Let $\varrho$ be a linear functional on $\mathscr{A}$. Then using (1.52) and (1.51) we get

$$
\begin{aligned}
& \varrho(a)=\varrho(e * a)=(\varrho * e)(a), \\
& \varrho(a)=\varrho(a * e)=(e * \varrho)(a),
\end{aligned}
$$


for any $a \in \mathscr{A}$. Therefore

$$
\varrho * e=e * \varrho=\varrho .
$$

In particular

$$
e * e=e
$$

\section{Elements of the Theory of Representations}

Let $G$ be a compact group, $K$ be a finite-dimensional complex vector space and $v$ be a representation of $G$ acting on $K$. The latter means that $v$ is a continuous map

$$
v: G \rightarrow B(K),
$$

and

$$
v(g) v\left(g^{\prime}\right)=v\left(g g^{\prime}\right),
$$

for any $g, g^{\prime} \in G$. Identifying the space of all continuous functions defined on $G$ taking values in $B(K)$ with $B(K) \otimes C(G)$, we have

$$
v \in B(K) \otimes C(G) \text {. }
$$

In the general case a representation of a compact matrix pseudogroup $G=(A, u)$ acting on $K$ is an element $v \in B(K) \otimes A$ satisfying a certain condition replacing (2.1). In order to formulate this condition we shall use a bilinear multiplication $(1)$ defined on $B(K) \otimes A$. It is introduced in the following way.

Let $A$ be a $C^{*}$-algebra and $K$ be a $f$-d.c. vector space. For any $v, w \in B(K) \otimes A$, we set

$$
v \oplus w=\sum_{i, j} m_{i} n_{j} \otimes v_{i} \otimes w_{j}
$$

where $m_{i}, n_{j} \in B(K) ; v_{i}, w_{j} \in A$ are such that $v=\sum_{i} m_{i} \otimes v_{i}$ and $w=\sum_{j} n_{j} \otimes w_{j}$. Clearly $v \oplus w$ is well defined [right-hand side of (2.2) is independent of the choice of $m_{i}, v_{i}, n_{j}$, and $w_{j}$ ] element of $B(K) \otimes A \otimes A$ and

$$
\text { (1) }:(B(K) \otimes A) \times(B(K) \otimes A) \rightarrow B(K) \otimes A \otimes A
$$

is a bilinear map.

Let

$$
i: B(K) \otimes A \rightarrow B(K) \otimes A \otimes A
$$

be an algebraic homomorphism such that $i(m \otimes a)=m \otimes I \otimes a$ for any $m \in B(K)$ and $a \in A$. One can easily check that

$$
v \oplus w=(v \otimes I) i(w)
$$

for any $v, w \in B(K) \otimes A$. If $K$ is a Hilbert space, then $B(K), B(K) \otimes A$ and $B(K) \otimes A \otimes A$ are $C^{*}$-algebras, $i$ is a $C^{*}$-homomorphism and (2.3) shows that $v \oplus w$ is unitary if $v$ and $w$ are unitary elements of $B(K) \otimes A$. 
If $A=C(G)$, then identifying $B(K) \otimes A$ and $B(K) \otimes A \otimes A$ with $C(G, B(K))$ and $C(G \times G, B(K))$ respectively, we have (for any $g, g^{\prime} \in G$ )

$$
\begin{aligned}
(v \oplus w)\left(g, g^{\prime}\right) & =\sum_{i, j} m_{i} n_{j} v_{i}(g) w_{j}\left(g^{\prime}\right) \\
& =\left(\sum_{i} m_{i} v_{i}(g)\right)\left(\sum_{j} n_{j} w_{j}\left(g^{\prime}\right)\right)=v(g) w\left(g^{\prime}\right) .
\end{aligned}
$$

Therefore (2.1) can be rewritten in the following "algebraic" form:

$$
v \oplus v=(\mathrm{id} \otimes \Phi) v,
$$

where $\Phi$ is the $C^{*}$-homomorphism $C(G) \rightarrow C(G) \otimes C(G)$ introduced by (1.18).

We turn back to the general case.

Definition 2.1. Let $G=(A, u)$ be a compact matrix pseudogroup, $\Phi$ be the comultiplication associated with $G, K$ be a finite-dimensional complex vector space and $v \in B(K) \otimes A$. We say that $v$ is a representation of $G$ acting on $K$ if

$$
v \oplus v=(\mathrm{id} \otimes \Phi) v .
$$

Let $G=(A, u)$, where $u=\left(u_{k l}\right)_{k l=1,2, \ldots, N} \in M_{N}(A)$ be a compact matrix pseudogroup. Identifying $M_{N}(A)$ with $M_{N} \otimes A$ we have

$$
u=\sum_{k l=1}^{N} m_{l k} \otimes u_{k l}
$$

where $\left(m_{l k}\right)_{l k=1,2, \ldots, N}$ is the system of matrix units: $m_{l k}$ is the $N \times N$ matrix having 1 on the corssing of $k^{\text {th }}$ row and $l^{\text {th }}$ column and zeroes in all other places. Using the well known rules of matrix calculus $\left(m_{l k} m_{s r}=\delta_{l r} m_{s k}\right.$ for any $\left.k, l, r, s=1,2, \ldots, N\right)$, and taking into account (1.2), we have

$$
\begin{aligned}
u \oplus u & =\sum_{k l r s} m_{l k} m_{s r} \otimes u_{k l} \otimes u_{r s} \\
& =\sum_{k r s} m_{s k} \otimes u_{k r} \otimes u_{r s}=(\mathrm{id} \otimes \Phi) u .
\end{aligned}
$$

It shows that $u$ is a representation of $G$ acting on $\mathbb{C}^{N}$ [remember that $\left.M_{N}=B\left(\mathbb{C}^{N}\right)\right]$. It is called the fundamental representation of $G$.

In many cases it is more convenient to work with the linear mapping $\hat{v}: K \rightarrow K \otimes A$ corresponding to $v \in B(K) \otimes A$ than with $v$ itself. $B(K, K \otimes A)$ and $B(K) \otimes A$ are canonically isomorphic: If an element $v \in B(K) \otimes A$ is given by

$$
v=\sum_{r=1}^{R} m_{r} \otimes v_{r}
$$

where $m_{1}, m_{2}, \ldots, m_{R} \in B(K)$ and $v_{1}, v_{2}, \ldots, v_{R} \in A$, then the action of $\hat{v}$ on an element $x \in K$ is defined by

$$
\hat{v} x=\sum_{r=1}^{R} m_{r} x \otimes v_{r}
$$

Assume that (2.4) is satisfied. Then [cf. (2.2) and (2.6)]

$$
\sum_{r r^{\prime}} m_{r} m_{r^{\prime}} \otimes v_{r} \otimes v_{r^{\prime}}=\sum_{r} m_{r} \otimes \Phi\left(v_{r}\right)
$$


and for any $x \in K$ we have

$$
\begin{aligned}
(\hat{v} \otimes \mathrm{id}) \hat{v} x & =\sum_{r^{\prime}} \hat{v} m_{r^{\prime}} x \otimes v_{r^{\prime}} \\
& =\sum_{r r^{\prime}} m_{r} m_{r^{\prime}} x \otimes v_{r} \otimes v_{r^{\prime}} \\
& =\sum_{r} m_{r} x \otimes \Phi\left(v_{r}\right)=(\mathrm{id} \otimes \Phi) \hat{v} x .
\end{aligned}
$$

It shows that the diagram



is commutative.

Conversely let $\hat{v}: K \rightarrow K \otimes A$ be a linear map such that the diagram (2.8) is commutative and $v$ be the element of $B(K) \otimes A$ corresponding to $\hat{v}$. Then $v$ is a representation of $G$ acting on $K$. The simple proof of this fact is left to the reader.

Let $v \in B(K) \otimes A$ and $A^{\prime}$ be the space of all continuous linear functionals defined on $A$. For any $\varrho \in A^{\prime}$, we set

$$
v_{\varrho}=(\mathrm{id} \otimes \varrho) v
$$

Then $v_{\varrho}$ is an operator acting on $K$. It depends linearly on $\varrho$. Taking into account the definition (2.2) we get immediately

$$
\left(\mathrm{id} \otimes \varrho \otimes \varrho^{\prime}\right)(v \oplus v)=v_{\varrho} v_{\varrho^{\prime}} \cdot
$$

On the other hand using the convolution product introduced by (1.50) we have:

$$
\left(\mathrm{id} \otimes \varrho \otimes \varrho^{\prime}\right)(\mathrm{id} \otimes \Phi) v=v_{\varrho * \varrho^{\prime}} .
$$

Therefore (2.4) is equivalent to the relation

$$
\left(\begin{array}{c}
\text { For any } \varrho, \varrho^{\prime} \in A^{\prime} \\
v_{\varrho_{*} \varrho^{\prime}}=v_{\varrho} v_{\varrho^{\prime}}
\end{array}\right) \text {. }
$$

In the representation theory an important role is played by intertwining operators.

Definition 2.2. Let $v$ and $w$ be representations of a compact matrix pseudogroup $G=(A, u)$ acting on a $f$-d.c. vector spaces $K_{v}$ and $K_{w}$ respectively. We say that an operator $s \in B\left(K_{v}, K_{w}\right)$ intertwines $v$ and $w$ if

$$
(s \otimes I) v=w(s \otimes I) .
$$

The set of all operators intertwining $v$ and $w$ will be denoted by $\operatorname{Mor}(v, w)$. Clearly $\operatorname{Mor}(v, w)$ is a linear subspace in $B\left(K_{v}, K_{w}\right)$. If $w=v$, then $\operatorname{Mor}(v, w)$ is a subalgebra of $B\left(K_{v}\right)$.

One can easily prove

Proposition 2.1. Let $v, w$ be representations of $G$. Then the following three statements are equivalent: 
1) $s \in \operatorname{Mor}(v, w)$.

2) The diagram

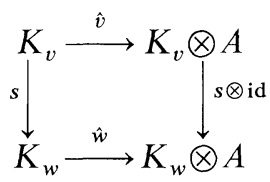

is commutative.

3) $s v_{\varrho}=w_{\varrho} s$ for any $\varrho \in A^{\prime}$.

Let $G=(A, u)$ be a compact matrix pseudogroup. We say that two representations $v$ and $w$ of $G$ are equivalent if there exists an invertible operator intertwining $v$ and $w$.

Let $v$ be a representation of $G$ acting on a $f$-d.c. vector space $K$ and $L$ be a subspace of $K$. We say that $L$ is $v$-invariant if $\hat{v}(L) \subset L \otimes A$. Then the element $\left.v\right|_{L} \in B(L) \otimes A$ corresponding to the restriction $\left.\hat{v}\right|_{L}: L \rightarrow L \otimes A$ is a representation of $G$ acting on $L$ and the embedding $L \rightarrow K$ intertwins $\left.v\right|_{L}$ and $\left.v \cdot v\right|_{L}$ is called a subrepresentation of $v$.

Let $v$ be a representation of $G$ acting on a $f$-d.c. vector space $K$. One can easily check that the subspaces

$$
\begin{aligned}
K_{0} & =\{x \in K: \hat{v} x=0\}, \\
K_{\text {inv }} & =\{x \in K: \hat{v} x=x \otimes I\},
\end{aligned}
$$

are $v$-invariant and $\left.v\right|_{K_{0}}=0,\left.v\right|_{K_{\text {inv }}}=I_{B\left(K_{\text {inv }}\right) \otimes A}$. The representation $v$ is called nondegenerate (completely degenerate respectively) if $K_{0}=\{0\}\left(K_{0}=K\right.$ respectively). An element $x \in K$ is said to be a $v$-invariant element if $x \in K_{\text {inv }}$. One can easily check that $x$ is a $v$-invariant element if and only if $v_{\varrho} x=\varrho(I) x$ for any $\varrho \in A^{\prime} . v$ is called trivial if $v=I_{B(K) \otimes I}$. Then $K_{\mathrm{inv}}=K$.

Let $f$ be a linear functional defined on $K$. We say that $f$ is a $v$-invariant functional if $f \in \operatorname{Mor}(v, \mathbb{1})$, where $\mathbb{1}$ denotes the trivial representation of $G$ acting on $\mathbb{C}$. Using Proposition 2.1 one can check that $f$ is a $v$-invariant functional if and only if $f \circ v_{\varrho}=\varrho(I) f$ for any $\varrho \in A^{\prime}$.

Let $v$ be a representation of $G$ acting on a $f$-d.c. vector space $K$. Clearly the whole $K$ and the zero-dimensional subspace $\{0\} \subset K$ are $v$-invariant. These subspaces are called trivial. The representation $v$ is called irreducible if there exists no non-trivial $v$-invariant subspace.

If $v$ and $w$ are representations of $G$ and $s \in \operatorname{Mor}(v, w)$, then the kernel of $s$ is $v$-invariant and the image of $s$ is $w$-invariant. Using this remark we get immediately

Proposition 2.2. Let $v$ be an irreducible representation of $G$. Then $\operatorname{Mor}(v, v)$ $=\{\lambda I: \lambda \in \mathbb{C}\}$.

Proposition 2.3. Let $v, w$ be irreducible representations of $G$. Then either $\operatorname{Mor}(v, w)$ $=\{0\}$ and $v$ is not equivalent to $w$, or $\operatorname{Mor}(v, w)=\{\lambda F: \lambda \in \mathbb{C}\}$ where $F$ is invertible and $v$ is equivalent to $w$.

For any finite-dimensional complex vector space $K$, the complex conjugate vector space will be denoted by $\bar{K}$. It means that $\bar{K}$ is the set of symbols $\bar{x}$, where $x$ 
runs over $K$ with the complex vector space structure introduced by the formulae $\bar{x}+\bar{y}=\overline{x+y}$ and $\lambda \bar{x}=\bar{\lambda} x(x, y \in K, \lambda \in \mathbb{C})$. We shall identify the second complex conjugate $\overline{\bar{K}}$ with $K$. Then the map

$$
K \ni x \rightarrow \bar{x} \in \bar{K}
$$

is an antilinear involution. For any $a \in B(K, L)$ (where $K$ and $L$ are $f$-d.c. vector spaces) we consider the operator $\bar{a} \in B(\bar{K}, \bar{L})$ introduced by the formula $\bar{a} \bar{x}=\overline{a x}$ $(x \in K)$. Evidently

$$
B(K, L) \ni a \rightarrow \bar{a} \in B(\bar{K}, \bar{L})
$$

is an antilinear involution. In the particular case $L=K$ this map is multiplicative: $\overline{a b}=\bar{a} \bar{b}$ for any $a, b \in B(K)$.

We know that the tensor product of antilinear maps is a well defined antilinear map. For any $v \in B(K) \otimes A$ we denote by $\bar{v}$ the element of $B(K) \otimes A$ obtained by applying ${ }^{-\otimes *}$ to $v: \bar{v}=v^{-\otimes *}$.

Assume that $v$ is a representation of a compact matrix pseudogroup $G=(A, u)$ acting on $K$. Then $\bar{v}$ is a representation of $G$ acting on $\bar{K}$. It is called complex conjugate to $v$. For instance [cf. (2.5)]

$$
\bar{u}=\sum_{k, l} \bar{m}_{l k} \otimes u_{k l}^{*}
$$

is the representation complex conjugate to the fundamental one.

Let $v$ and $w$ be representations of $G$ and $s \in \operatorname{Mor}(v, w)$. Then $\bar{s} \in \operatorname{Mor}(\bar{v}, \bar{w})$. In particular $\bar{v}$ and $\bar{w}$ are equivalent if and only if $v$ and $w$ are equivalent.

Let $K_{1}$ and $K_{2}$ be $f$-d.c. vector spaces and

$$
\begin{aligned}
& K_{1} \stackrel{i_{1}}{\longrightarrow} K_{1} \oplus K_{2} \stackrel{\pi_{1}}{\longrightarrow} K_{1} \\
& K_{2} \stackrel{i_{2}}{\longrightarrow} K_{1} \oplus K_{2} \stackrel{\pi_{2}}{\longrightarrow} K_{2}
\end{aligned}
$$

be canonical embeddings and projections. For any $v_{1} \in B\left(K_{1}\right) \otimes A$ and $v_{2} \in B\left(K_{2}\right) \otimes A$, we set

$$
v_{1} \oplus v_{2}=\left(i_{1} \otimes I\right) v_{1}\left(\pi_{1} \otimes I\right)+\left(i_{2} \otimes I\right) v_{2}\left(\pi_{2} \otimes I\right) .
$$

Clearly $v_{1} \oplus v_{2} \in B\left(K_{1} \oplus K_{2}\right) \otimes A$.

Assume that $v_{1}$ and $v_{2}$ are representations of a compact matrix pseudogroup $G=(A, u)$ acting on $K_{1}$ and $K_{2}$ respectively. Then $v_{1} \oplus v_{2}$ is a representation of $G$ acting on $K_{1} \oplus K_{2}$ and

$$
\begin{array}{ll}
i_{1} \in \operatorname{Mor}\left(v_{1}, v_{1} \oplus v_{2}\right), & \pi_{1} \in \operatorname{Mor}\left(v_{1} \oplus v_{2}, v_{1}\right), \\
i_{2} \in \operatorname{Mor}\left(v_{2}, v_{1} \oplus v_{2}\right), & \pi_{2} \in \operatorname{Mor}\left(v_{1} \oplus v_{2}, v_{2}\right) .
\end{array}
$$

The representation $v_{1} \oplus v_{2}$ is called the direct sum of $v_{1}$ and $v_{2}$. In the similar way one can introduce the direct sum of any finite number of representations.

Let $v_{1}, v_{2}, w_{1}, w_{2}$ be representations of $G$. Assume that

$$
s_{1} \in \operatorname{Mor}\left(v_{1}, w_{1}\right) \text { and } s_{2} \in \operatorname{Mor}\left(v_{2}, w_{2}\right) .
$$

Then $s_{1} \oplus s_{2}$ intertwines $v_{1} \oplus v_{2}$ with $w_{1} \oplus w_{2}$. In particular if $v_{1}$ is equivalent to $w_{1}$ and $v_{2}$ is equivalent to $w_{2}$, then $v_{1} \oplus v_{2}$ is equivalent to $w_{1} \oplus w_{2}$. 
Let $K, L$ be $f$-d.c. vector spaces, $v \in B(K) \otimes A$ and $w \in B(L) \otimes A$. Then there exist $m_{1}, m_{2}, \ldots, m_{R} \in B(K), n_{1}, n_{2}, \ldots, n_{S} \in B(L)$ and $v_{1}, v_{2}, \ldots, v_{R}, w_{1}, w_{2}, \ldots, w_{S} \in A$ such that

$$
\begin{gathered}
v=\sum_{r} m_{r} \otimes v_{r}, \\
w=\sum_{s} n_{s} \otimes w_{s} .
\end{gathered}
$$

We set

$$
v \oplus w=\sum_{r, s} m_{r} \otimes n_{s} \otimes v_{r} w_{s}
$$

Clearly $v \oplus w$ is a well defined [right-hand side of (2.15) depends only on $v$ and $w$, it is independent of the particular choice of $m_{r}, v_{r}, n_{s}, w_{s}$ entering (2.13) and (2.14)] element of $B(K) \otimes B(L) \otimes A$ and identifying $B(K) \otimes B(L)$ with $B(K \otimes L)$, we introduce bilinear map

$$
\bigoplus:(B(K) \otimes A) \times(B(L) \otimes A) \rightarrow B(K \otimes L) \otimes A .
$$

Let $m \in B(K)$ and $n \in B(L)$. Using definition (2.15), one can immediately check that

$$
\begin{aligned}
& (v \oplus w)(m \otimes n \otimes I)=[v(m \otimes I)] \oplus[w(n \otimes I)], \\
& (m \otimes n \otimes I)(v \oplus w)=[(m \otimes I) v] \oplus[(n \otimes I) w] .
\end{aligned}
$$

Let us notice that for any $v, v^{\prime} \in B(K) \otimes A$ and $w, w^{\prime} \in B(L) \otimes A$, we have

$$
\left(v \oplus v^{\prime}\right) \oplus\left(w \oplus w^{\prime}\right)=(v \oplus w) \oplus\left(v^{\prime} \oplus w^{\prime}\right) \text {. }
$$

Indeed in the simplest case $v=m_{1} \otimes v_{1}, v^{\prime}=m_{1}^{\prime} \otimes v_{1}^{\prime}, w=n_{1} \otimes w_{1}, w^{\prime}=n_{1}^{\prime} \otimes w_{1}^{\prime}$ [where $m_{1}, m_{1}^{\prime} \in B(K), n_{1}, n_{1}^{\prime} \in B(L), v_{1}, v_{1}^{\prime}, w_{1}, w_{1}^{\prime} \in A$ ] we have

$$
v \oplus v^{\prime}=m_{1} m_{1}^{\prime} \otimes v_{1} \otimes v_{1}^{\prime}, \quad w \oplus w^{\prime}=n_{1} n_{1}^{\prime} \otimes w_{1} \otimes w_{1}^{\prime},
$$

and

$$
\left(v \oplus v^{\prime}\right) \oplus\left(w \oplus w^{\prime}\right)=m_{1} m_{1}^{\prime} \otimes n_{1} n_{1}^{\prime} \otimes v_{1} w_{1} \otimes v_{1}^{\prime} w_{1}^{\prime} .
$$

On the other hand

$$
\begin{gathered}
v \oplus w=m_{1} \otimes n_{1} \otimes v_{1} w_{1}, \quad v^{\prime} \oplus w^{\prime}=m_{1}^{\prime} \otimes n_{1}^{\prime} \otimes v_{1}^{\prime} w_{1}^{\prime}, \\
(v \oplus w) \oplus\left(v^{\prime} \oslash w^{\prime}\right)=m_{1} m_{1}^{\prime} \otimes n_{1} n_{1}^{\prime} \otimes v_{1} w_{1} \otimes v_{1}^{\prime} w_{1}^{\prime}
\end{gathered}
$$

and (2.18) follows. Moreover taking into account (2.15) and multiplicativity of $\Phi$, one can easily check that

$$
(\mathrm{id} \otimes \Phi)(v \oplus w)=(\mathrm{id} \otimes \Phi) v \oplus(\mathrm{id} \otimes \Phi) w .
$$

Assume now that $v$ and $w$ are representations of a compact matrix pseudogroup $G=(A, u)$ acting on $K$ and $L$ respectively. It follows immediately from (2.18) and (2.19) that $v \bigoplus w$ is a representation of $G$ acting on $K \otimes L$. This representation is called the tensor product of $v$ and $w$.

Let $v, v^{\prime}, w, w^{\prime}$ be representations of $G, s \in \operatorname{Mor}\left(v, v^{\prime}\right)$ and $r \in \operatorname{Mor}\left(w, w^{\prime}\right)$. Using (2.16) and (2.17) one can easily check that $s \otimes r \in \operatorname{Mor}\left(v \oplus w, v^{\prime} \oplus w^{\prime}\right)$. In particular the equivalence class of $v \oplus w$ depends only on equivalence classes of $v$ and $w$. 
Let $v_{1}, v_{2}, v_{3}$ be representations of $G$ acting on $f$-d.c. vector spaces $K_{1}, K_{2}, K_{3}$ respectively. Identifying $\left(K_{1} \otimes K_{2}\right) \otimes K_{3}$ with $K_{1} \otimes\left(K_{2} \otimes K_{3}\right)$ and using (2.15), one easily verifies the associativity law:

$$
\left(v_{1} \oplus v_{2}\right) \oplus v_{3}=v_{1} \oplus\left(v_{2} \oplus v_{3}\right) .
$$

In the group theory the tensor product of representations is commutative. Identifying $K \otimes L$ with $L \otimes K$ we had $v \oplus w=w \oplus v$ for any representations $v$ and $w$ acting on $K$ and $L$ respectively. For pseudogroups this is no longer the case.

Let $v$ and $w$ be representations of a compact matrix pseudogroup $G$ acting on $f$-d.c. vector spaces $K$ and $L$ respectively. We say that $v$ commutes with $w$ if the operator $\sigma_{K L}$ interchanging $K$ and $L\left(\sigma_{K L}: K \otimes L \rightarrow L \otimes K\right.$ and $\sigma_{K L}(x \otimes y)=y \otimes x$ for any $x \in K$ and $y \in L)$ intertwines $v \oplus w$ with $w \oplus v$. We have the following

Proposition 2.4. Let $G=\left(A,\left(u_{k l}\right)_{k l=1,2, \ldots, N}\right)$ be a compact matrix pseudogroup. The following three conditions are equivalent:

1. $A$ is commutative. [In this case $G$ is a usual compact group of matrices (cf. Theorem 1.5).]

2. The fundamental representation $u$ commutes with $u$ and $\bar{u}$.

3. Any two representations of $G$ commute.

Proof. We shall use the following obvious formula:

$$
\sigma_{K L}(n \otimes m)=(m \otimes n) \sigma_{K L}
$$

for any $n \in B(K)$ and $m \in B(L)$.

Assume that $v \in B(K) \otimes A$ and $w \in B(L) \otimes A$ given by (2.13) and (2.14) are representations of $G$. Then

$$
\begin{aligned}
(v \oplus w)\left(\sigma_{K L} \otimes I\right) & =\sum_{r, s}\left(m_{r} \otimes n_{s}\right) \sigma_{K L} \otimes v_{r} w_{s}, \\
\left(\sigma_{K L} \otimes I\right)(w \oplus v) & =\sum_{r, s} \sigma_{K L}\left(n_{s} \otimes m_{r}\right) \otimes w_{s} v_{r} \\
& =\sum_{r, s}\left(m_{r} \otimes n_{s}\right) \sigma_{K L} \otimes w_{s} v_{r} .
\end{aligned}
$$

The representation $v$ commutes with $w$ if and only if the two expressions coincide, i.e. if and only if

$$
\sum_{r, s}\left(m_{r} \otimes n_{s}\right) \sigma_{K L} \otimes\left[v_{r}, w_{s}\right]=0 .
$$

If $A$ is commutative, then (2.22) is fulfilled and $v$ commutes with $w$. This way we proved that $1 \Rightarrow 3$. Implication $3 \Rightarrow 2$ is obvious.

Assume now that $u$ commutes with $u$ and $\bar{u}$. Using condition (2.22) we obtain [cf. (2.5) and (2.12)].

$$
\begin{aligned}
& \sum_{\substack{r r^{\prime} \\
s s^{\prime}}}\left(m_{r r^{\prime}} \otimes m_{s s^{\prime}}\right) \sigma_{K K} \otimes\left[u_{r^{\prime} r}, u_{s^{\prime} s}\right]=0, \\
& \sum_{\substack{r r^{\prime} \\
s s^{\prime}}}\left(m_{r r^{\prime}} \otimes \bar{m}_{s s^{\prime}}\right) \sigma_{K \tilde{K}} \otimes\left[u_{r^{\prime} r}, u_{s^{\prime} s} *\right]=0,
\end{aligned}
$$

where now $K=\mathbb{C}^{N}$ is the carrier vector space of the fundamental representation. Since the matrices $m_{r r^{\prime}}\left(r, r^{\prime}=1,2, \ldots, N\right)$ are linearly independent, the above 
equations mean that

$$
\left[u_{r^{\prime} r}, u_{s^{\prime} s}\right]=\left[u_{r^{\prime} r}, u_{s^{\prime} s}^{*}\right]=0
$$

for all $r^{\prime}, r, s^{\prime}, s=1,2, \ldots, N$. Therefore the *-algebra $\mathscr{A}$ (generated by matrix elements of $u$ ) is commutative and (cf. Condition 1 of Definition 1.1) Statement 1 follows. Q.E.D.

Let $\mathscr{R}_{G}$ be the smallest class of representations of $G$ containing the fundamental representation and closed under direct sums, tensor products, complex conjugation and passing to a subrepresentation and to an equivalent representation. It turns out that the class $\mathscr{R}_{G}$ is rich in the following sense

Proposition 2.5. Let $G=(A, u)$ be a compact matrix pseudogroup and $\mathscr{A}$ be the *-subalgebra of A generated by matrix elements of $u$. Then any element $a \in \mathscr{A}$ can be written in the form

$$
a=(\tau \otimes \mathrm{id}) v,
$$

where $v \in \mathscr{R}_{G}$, v acts on a $f$-d.c. vector space $K$ and $\tau$ is a linear functional defined on $B(K)$.

Proof. Let $\mathscr{A}_{1}$ be the set of all elements $a \in \mathscr{A}$ which can be written in the form (2.23). We have to show that $\mathscr{A}_{1}=\mathscr{A}$. Setting $v=u$ we see that all matrix elements of $u$ belong to $\mathscr{A}_{1}$. Therefore it is sufficient to show that $\mathscr{A}_{1}$ is a ${ }^{*}$-subalgebra of $A$. The latter follows immediately from the following obvious formulae:

$$
\begin{aligned}
\lambda(\tau \otimes \mathrm{id}) v & =(\lambda \tau \otimes \mathrm{id}) v, \\
(\tau \otimes \mathrm{id}) v+\left(\tau^{\prime} \otimes \mathrm{id}\right) v^{\prime} & =\left(\left(\tau \oplus \tau^{\prime}\right) \otimes \mathrm{id}\right)\left(v \oplus v^{\prime}\right), \\
{[(\tau \otimes \mathrm{id}) v]\left[\left(\tau^{\prime} \otimes \mathrm{id}\right) v^{\prime}\right] } & =\left(\tau \otimes \tau^{\prime} \otimes \mathrm{id}\right)\left(v \oplus v^{\prime}\right), \\
{[(\tau \otimes \mathrm{id}) v]^{*} } & =(\bar{\tau} \otimes \mathrm{id}) \bar{v},
\end{aligned}
$$

where $\lambda \in \mathbb{C} ; v$ and $v^{\prime}$ are representations of $G$ acting on $K$ and $L$ respectively; $\tau$ and $\tau^{\prime}$ are linear functionals defined on $B(K)$ and $B(L)$ respectively; $\tau \oplus \tau^{\prime}$ is the linear functional defined on $B(K \oplus L)$ such that $\left(\tau \oplus \tau^{\prime}\right)\left(m \oplus m^{\prime}\right)=\tau(m)+\tau^{\prime}\left(m^{\prime}\right)$ for any $m \in B(K)$ and $m^{\prime} \in B(L)$ and finally $\bar{\tau}$ is a linear functional defined on $B(\bar{K})$ such that $\bar{\tau}(\bar{m})=\overline{\tau(m)}(m \in B(K))$. Q.E.D.

Let $\left(a_{n}\right)_{n \in \mathbb{N}}$ be a sequence of elements of a topological vector space. We say that the sequence converges in the Cesàro sense and that $g$ is the Cesàro limit of $\left(a_{n}\right): g=C$ - $\lim _{n \rightarrow \infty} a_{n}$ if the sequence $\left(b_{n}\right)_{n \in \mathbb{N}}$ of arithmetic means $\left(b_{n}=\frac{1}{n} \sum_{k=1}^{n} a_{k}\right)$ converges to $g$ in the usual sense.

We shall use the following

Lemma 2.6. Let $K$ be a $f$-d.c. vector space and $t \in B(K)$. Assume that the sequence of iterations $\left(t^{n}\right)_{n \in \mathbb{N}}$ is bounded. Then there exists the Cesàro limit

$$
t_{\infty}=\underset{n \rightarrow \infty}{C-\lim _{n}} t^{n}
$$

and $t_{\infty}$ is a projection onto $\{x \in K: t x=x\}$. 
Proof. Using if necessary Jordan decomposition one may assume that $t=\lambda I_{B(K)}+N$, where $\lambda \in \mathbb{C}$ and $N$ is nilpotent. The sequence $\left(t^{n}\right)_{n \in \mathbb{N}}$ is bounded in the following two cases:

I. $|\lambda|<1$. Then $C-\lim t^{n}=\lim t^{n}=0$

II. $|\lambda|=1$ and $N=0$. Then

$$
C-\lim _{n \rightarrow \infty} t^{n}=\left\{\begin{array}{ll}
I_{B(K)} & \text { if } \lambda=1 \\
0 & \text { otherwise. }
\end{array}\right. \text { Q.E.D. }
$$

For any state $\varrho$ [i.e. positive linear functional such that $\varrho(I)=1]$ defined on $A$ we denote $\varrho^{* n}=\varrho * \varrho * \ldots * \varrho(n$-factors $)$ the $n^{\text {th }}$ convolution power of $\varrho$. Clearly $\varrho^{* n}$ is a state of $A$. We shall prove

Proposition 2.7. For any $a \in A$ and any state $\varrho$ of $A$ there exists the Cesaro limit,

$$
h_{\varrho}(a)=\underset{n \rightarrow \infty}{C-\lim _{i}} \varrho^{* n}(a) .
$$

$h_{\varrho}$ is a state of $A$. Moreover we have

$$
\varrho * h_{\varrho}=h_{\varrho} * \varrho=h_{\varrho} .
$$

Proof. The sequence of states $\left(\varrho^{* n}\right)$ is norm bounded and $\mathscr{A}$ is dense in $A$. Therefore it is sufficient to prove the existence of limit (2.24) for all $a \in \mathscr{A}$. By virtue of Proposition 2.5 one may assume that $a$ is of the form (2.23). Then using the notation introduced by (2.9) we have [cf. (2.10)]

$$
\varrho^{* n}(a)=\left(\tau \otimes \varrho^{* n}\right) v=\tau\left(v_{\varrho^{* n}}\right)=\tau\left(v_{\varrho}^{n}\right)
$$

and the existence of the Cesàro limit (2.24) follows immediately from Lemma 2.6 [sequence $\left(\varrho^{* n}\right)_{n \in \mathbb{N}}$ is norm bounded and $v_{\varrho}^{n}=\left(\mathrm{id} \otimes \varrho^{* n}\right) v$ ]. Clearly the linear functional $h_{\varrho}$ introduced by (2.24) is positive and normalized. In order to prove (2.25) it is sufficient to set $n \rightarrow \infty$ in the following relation

$$
\varrho * \varrho^{* n}=\varrho^{* n} * \varrho=\varrho^{*(n+1)} \text {. Q.E.D. }
$$

\section{Contragradient Representations}

This section is devoted to the concept of the representation contragradient to a given one. As we shall see later this concept plays the fundamental role in our approach to pseudogroups.

Throughout this section $G=(A, u)$ is a compact matrix pseudogroup, $\Phi$ is the comultiplication and $\kappa$ is the coinverse associated with $G, \mathscr{A}$ is the *-subalgebra of $A$ generated by matrix elements of $u$ and $e: \mathscr{A} \rightarrow \mathbb{C}$ is the ${ }^{*}$-character introduced in Proposition 1.8.

In the theory of groups the definition of the representation contragradient to a given one involves the involution $g \rightarrow g^{-1}$. Unfortunately the algebraic counterpart of this involution, i.e. the coinverse map $\kappa$ is not defined on the whole $A$. Therefore we have to limit our considerations to the representations with "matrix elements" belonging to $\mathscr{A}$. Such representations are called smooth. 
It turns out that the class of smooth representations is sufficiently large. In fact the representations which are not smooth (if they exist) have pathological properties. See the last sections for the details.

Let $K$ be a finite dimensional complex vector space and $v \in B(K) \otimes A$ be a representation of $G$ acting on $K$. We say that $v$ is a smooth representation if $v \in B(K) \otimes \mathscr{A}$.

According to (2.5) the fundamental representation is smooth. One can easily check that complex conjugation, direct sum and tensor product applied to smooth representations produce smooth representations. Moreover any subrepresentation of a smooth representation is smooth. Therefore all representations in $\mathscr{R}_{G}$ are smooth.

If $v$ is a smooth representation then (2.9) is meaningful for any linear functional $\varrho$ defined on $\mathscr{A}$. Moreover in this case the product formula (2.10) holds for all linear functionals $\varrho, \varrho^{\prime}$ defined on $\mathscr{A}$ [cf. the remark after formula (1.51)]. No continuity assumption is required

Proposition 3.1. Let $v$ be a smooth representation of $G$ acting on a finite dimensional complex vector space $K$. Then

$$
K=K_{0} \oplus K_{1},
$$

where $K_{0}$ and $K_{1}$ are $v$-invariant subspaces, $\left.v\right|_{K_{0}}$ is completely degenerate representation and $\left.v\right|_{K_{1}}$ is nondegenerate.

Proof. We use the functional $e$ introduced in Proposition 1.8. Let $v_{e}=(\mathrm{id} \otimes e) v[\mathrm{cf}$. (2.9)]. By virtue of (1.54) and (2.10) $v_{e}^{2}=v_{e}$, i.e. $v_{e}$ is a projection acting on $K$. Therefore

$$
K=K_{0} \oplus K_{1},
$$

where $K_{0}=\operatorname{ker} v_{e}$ and $K_{1}=$ range $v_{e}$. It follows immediately from (1.53) and (2.10) that $v_{e} v_{\varrho}=v_{\varrho} v_{e}$ for any $\varrho \in A^{\prime}$. Therefore (cf. Proposition 2.1) $v_{e} \in \operatorname{Mor}(v, v)$ and $K_{0}$ and $K_{1}$ are $v$-invariant.

Assume that a vector $x \in K$ is killed by the representation $v$. It means that $\hat{v} x=0$. Then $v_{\varrho} x=0$ for any $\varrho \in \mathscr{A}^{\prime}$. In particular $v_{e} x=0$ and $x \in K_{0}$. Conversely if $x \in K_{0}$ then using (1.53) and (2.10) we have $v_{\varrho} x=v_{\varrho * e} x=v_{\varrho} v_{e} x=0$ for any $\varrho \in A^{\prime}$. It means that $\hat{v} x=0$. This way we proved that $K_{0}$ is the space of all vectors killed by $v$. Therefore $v$ restricted to $K_{0}$ is completely degenerate and $v$ restricted to $K_{1}$ is nondegenerate (no non-zero vector belonging to $K_{1}$ is killed by $v$ ). Q.E.D.

The following proposition gives nice criteria distinguishing non-degenerate representations.

Proposition 3.2. Let $v$ be a smooth representation of $G$ acting on $K$. Then the following four conditions are equivalent:

1. $v$ is non-degenerate,

2. $v_{e}=I_{B(K)}$,

3. $v$ is an invertible element of $B(K) \otimes A$ and

$$
v^{-1}=(\mathrm{id} \otimes \kappa) v,
$$

4. $v$ has a left inverse in $B(K) \otimes A$. 
Proof. $1 \Rightarrow 2$. If $v$ is non-degenerate then $K_{0}$ in the decomposition (3.1) vanishes. It means that $\operatorname{ker} v_{e}=\{0\}$ and $v_{e} x=x$ for any $x \in K$.

$2 \Rightarrow 3$. Let

$$
v=\sum m_{r} \otimes v_{r},
$$

where $m_{r} \in B(K), v_{r} \in \mathscr{A}$ and $r=1,2, \ldots, R$. Then $v_{e}=\sum m_{r} e\left(v_{r}\right)$. Formula (2.4) means that

$$
\sum_{r} m_{r} \otimes \Phi\left(v_{r}\right)=\sum_{r, s} m_{r} m_{s} \otimes v_{r} \otimes v_{s} .
$$

Applying to the both sides of the above equation the mapping $\mathrm{id} \otimes m(\kappa \otimes \mathrm{id})$ [where $m$ is the multiplication map (1.35)] and using (1.38) we obtain

$$
\sum_{r} m_{r} \otimes e\left(v_{r}\right) I=\sum_{r, s} m_{r} m_{s} \otimes \kappa\left(v_{r}\right) v_{s} .
$$

By virtue of (3.3) this formula can be rewritten in the following compact way

$$
v_{e} \otimes I=[(\mathrm{id} \otimes \kappa) v] v .
$$

Similarly applying to the both sides of (3.4) the mapping $\mathrm{id} \otimes m(\mathrm{id} \otimes \kappa)$ and using (1.39) we get

$$
v_{e} \otimes I=v[(\mathrm{id} \otimes \kappa) v] .
$$

Now the implication $2 \Rightarrow 3$ is trivial

$3 \Rightarrow 4$. Obvious.

$4 \Rightarrow 1$. Let $v^{\prime}$ be a left inverse of $v: v^{\prime} v=I_{B(K)} \otimes A$. Then using the obvious action of elements of $B(K) \otimes A$ on $K \otimes A$, we have $v^{\prime} \hat{v} x=x \otimes I$ for any $x \in K$. Therefore $\hat{v} x=0$ implies $x=0$ and $v$ is non-degenerate. Q.E.D.

We know that $e$ is a $*$-character. Using this fact one can easily prove that $(v \oplus w)_{e}=v_{e} \oplus w_{e},(v \oplus w)_{e}=v_{e} \otimes w_{e}$ and $\bar{v}_{e}=\left(v_{e}\right)^{-}$. Therefore direct sum, tensor product and complex conjugation applied to smooth non-degenerate representations produce nondegenerate representations. Formula (1.37) shows that the fundamental representation is non-degenerate. It proves that all representations in $\mathscr{R}_{G}$ are non-degenerate.

For any finite-dimensional complex vector space $K$ the space dual to $K$ (i.e. the space of all linear functionals defined on $K$ ) will be denoted by $K^{\prime}$. For any $x \in K$ and $x^{\prime} \in K^{\prime}$ the value of the functional $x^{\prime}$ at the point $x$ will be denoted by $\left\langle x^{\prime}, x\right\rangle$. Setting $\left\langle x, x^{\prime}\right\rangle=\left\langle x^{\prime}, x\right\rangle$ we identify the second dual $K^{\prime \prime}$ with $K$.

For any $a \in B(K, L)$ (where $K, L$ are finite-dimensional complex vector spaces) we consider the transposed linear mapping $a^{T} \in B\left(L^{\prime}, K^{\prime}\right)$ such that $\left\langle a^{T} y^{\prime}, x\right\rangle$ $=\left\langle y^{\prime}, a x\right\rangle$ for all $y^{\prime} \in L^{\prime}$ and $x \in K$. The transposition

$$
T: B(K, L) \rightarrow B\left(L^{\prime}, K^{\prime}\right)
$$

is a linear involution. Moreover

$$
(a b)^{T}=b^{T} a^{T}
$$

for any $a \in B(K, L)$ and $b \in B(H, K)(H, K, L$ are $f$-d.c. vector spaces). 
For any $y^{\prime} \in L^{\prime}$ and $x^{\prime} \in K^{\prime}, y^{\prime} \otimes x^{\prime}$ will denote the linear functional defined on $K \otimes L$ such that

$$
\left\langle y^{\prime} \otimes x^{\prime}, x \otimes y\right\rangle=\left\langle x^{\prime}, x\right\rangle\left\langle y^{\prime}, y\right\rangle
$$

for any $x \in K$ and $y \in L$. In other words we identify $L^{\prime} \otimes K^{\prime}$ with $(K \otimes L)^{\prime}$. Let us notice that

$$
(a \otimes b)^{T}=b^{T} \otimes a^{T}
$$

for any $a \in B(K)$ and $b \in B(L)$. Indeed for any $y^{\prime} \in L^{\prime}, x^{\prime} \in K^{\prime}, x \in K$, and $y \in L$ we have

$$
\begin{aligned}
& \left\langle(a \otimes b)^{T}\left(y^{\prime} \otimes x^{\prime}\right), x \otimes y\right\rangle=\left\langle y^{\prime} \otimes x^{\prime},(a \otimes b)(x \otimes y)\right\rangle \\
& \quad=\left\langle y^{\prime} \otimes x^{\prime}, a x \otimes b y\right\rangle=\left\langle x^{\prime}, a x\right\rangle\left\langle y^{\prime}, b y\right\rangle=\left\langle a^{T} x^{\prime}, x\right\rangle\left\langle b^{T} y^{\prime}, y\right\rangle \\
& \quad=\left\langle b^{T} y^{\prime} \otimes a^{T} x^{\prime}, x \otimes y\right\rangle=\left\langle\left(b^{T} \otimes a^{T}\right)\left(y^{\prime} \otimes x^{\prime}\right), x \otimes y\right\rangle .
\end{aligned}
$$

Let $K$ and $L$ be $f$-d.c. vector spaces. For any $y_{i} \in L, x_{i}^{\prime} \in K^{\prime}$ (where $i=1,2, \ldots, S$ ) and any $x \in K$ we set

$$
\left(\sum_{i} y_{i} \otimes x_{i}^{\prime}\right) x=\sum_{i}\left\langle x_{i}^{\prime}, x\right\rangle y_{i}
$$

This way we identify $L \otimes K^{\prime}$ with $B(K, L)$. Let us notice that

$$
\left(n \otimes m^{T}\right) a=n a m
$$

for any $n \in B(L), m \in B(K)$, and $a \in B(K, L)$. Indeed if $a=\sum_{i} y_{i} \otimes x_{i}^{\prime}$, then

$$
\left(n \otimes m^{T}\right) a=\sum_{i} n y_{i} \otimes m^{T} x_{i}^{\prime}
$$

and for any $x \in K$, we have

$$
\begin{aligned}
\left(\left(n \otimes m^{T}\right) a\right) x & =\sum_{i}\left\langle m^{T} x_{i}^{\prime}, x\right\rangle n y_{i}=\sum_{i}\left\langle x_{i}^{\prime}, m x\right\rangle n y_{i} \\
& =n\left(\sum_{i} y_{i} \otimes x_{i}^{\prime}\right) m x=n a m x .
\end{aligned}
$$

Let $K$ and $L$ be $f$-d.c. vector spaces. We have the chain of natural identifications:

$$
B(L, K)^{\prime}=\left(K \otimes L^{\prime}\right)^{\prime}=L^{\prime \prime} \otimes K^{\prime}=L \otimes K^{\prime}=B(K, L) .
$$

Using (3.6) and (3.8) on can easily check that the duality between $B(L, K)$ and $B(K, L)$ is given by the formula

$$
\langle b, a\rangle=\operatorname{Tr} b a
$$

for any $b \in B(L, K)$ and $a \in B(K, L)$.

Now let $\mathscr{A}$ be a $*$-subalgebra of $A$ generated by matrix elements of $u$ and $\kappa: \mathscr{A} \rightarrow \mathscr{A}$ be the coinverse associated with $G$. For any $v \in B(K, L) \otimes \mathscr{A}(K, L$ are $f$-d.c. vector spaces) we consider an element $v^{c} \in B\left(L^{\prime}, K^{\prime}\right) \otimes \mathscr{A}$ introduced by the formula

$$
v^{c}=(T \otimes \kappa) v .
$$


Taking into account (3.5) one can easily check that

$$
\begin{aligned}
(w(s \otimes I))^{c} & =\left(s^{T} \otimes I\right) w^{c}, \\
((s \otimes I) v)^{c} & =v^{c}\left(s^{T} \otimes I\right)
\end{aligned}
$$

for any $v \in B(K) \otimes \mathscr{A}, w \in B(L) \otimes \mathscr{A}$ and $s \in B(K, L)$. Moreover using (3.7) and the antimultiplicativity of $\kappa$ we see that

$$
(v \oplus w)^{c}=w^{c} \oplus v^{c}
$$

for any $v \in B(K) \otimes \mathscr{A}$ and $w \in B(L) \otimes \mathscr{A}$.

Proposition 3.3. Let $v$ be a smooth representation of $G$ acting on a $f$-d.c. vector space $K$. Then $v^{c}$ is a representation of $G$ acting on $K^{\prime}$.

Proof. Using (1.47) we compute

$$
\begin{aligned}
(\mathrm{id} \otimes \Phi) v^{c} & =(T \otimes \Phi \circ \kappa) v=\left(T \otimes \sigma_{A}(\kappa \otimes \kappa) \Phi\right) v \\
& =\left(T \otimes \sigma_{A}(\kappa \otimes \kappa)\right) v \oplus v \\
& =\left(T \otimes \sigma_{A}\right)(\mathrm{id} \otimes \kappa) v \oplus(\mathrm{id} \otimes \kappa) v \\
& =(T \otimes \kappa) v \oplus(T \otimes \kappa) v=v^{c} \oplus v^{c},
\end{aligned}
$$

where $\sigma_{A}$ denotes the flip automorphism of $A \otimes A$ and the last but one equality follows easily from (3.5) and (2.2). Q.E.D.

The representation $v^{c}$ is said to be contragradient to the representation $v$. Clearly $v^{c}$ is smooth.

Let $v$ and $w$ be representations of $G$ and $s \in \operatorname{Mor}(v, w)$. Then using (3.12) and (3.13) one can easily show that $s^{T} \in \operatorname{Mor}\left(w^{c}, v^{c}\right)$. In particular $v^{c}$ and $w^{c}$ are equivalent if and only if $v$ and $w$ are equivalent. Moreover $v^{c}$ is irreducible if and only if $v$ is irreducible.

Let $v$ be a smooth representation of $G$ acting on a $f$-d.c. vector space $K$ and $x^{\prime} \in K^{\prime}$. One can easily verify that $x^{\prime}$ is a $v$-invariant functional if and only if $x^{\prime}$ is a $v^{c}$-invariant element.

The Haar measure (cf. Proposition 4.3) will provide us with a powerful tool for the investigation of $v$-invariant elements and functionals. The following proposition shows that the same techniques can be used for the investigation of intertwining operators. It uses many of the identifications described above: In Statement $1 B(K, L)=L \otimes K^{\prime}$, in Statement $2 B(L, K)=\left(L \otimes K^{\prime}\right)^{\prime}$, in Statement 3 $B\left(L^{\prime}, K^{\prime}\right)=K^{\prime} \otimes L$ and finally in Statement $4 B\left(K^{\prime}, L^{\prime}\right)=\left(K^{\prime} \otimes L\right)$.

Proposition 3.4. Let $v$ and $w$ be representation of $G$ acting on $f$-d.c. vector spaces $K$ and $L$ respectively, $a \in B(K, L)$ and $b \in B(L, K)$. Assume that $v$ is smooth and nondegenerate. Then

1. $a \in \operatorname{Mor}(v, w)$ if and only if $a$ is $a\left(w \oplus v^{c}\right)$-invariant element of $L \otimes K^{\prime}$,

2. $b \in \operatorname{Mor}\left(w, v^{c c}\right)$ if and only if $b$ is $a\left(w \oplus v^{c}\right)$-invariant functional on $L \otimes K^{\prime}$,

3. $a \in \operatorname{Mor}\left(v^{c c}, w\right)$ if and only if $a^{T}$ is a $\left(v^{c} \triangleright w\right)$-invariant element of $K^{\prime} \otimes L$,

4. $b \in \operatorname{Mor}(w, v)$ if and only if $b^{T}$ is a $\left(v^{c} \oplus w\right)$-invariant functional on $K^{\prime} \otimes L$. 
Proof. Let

$$
v=\sum_{i} m_{i} \otimes v_{i}, \quad w=\sum_{j} n_{j} \otimes w_{j},
$$

where $m_{i} \in B(K), v_{i} \in \mathscr{A}, n_{j} \in B(L)$, and $w_{j} \in A$. Then

$$
\left(w \oplus v^{c}\right)=\sum_{i, j} n_{j} \otimes m_{i}^{T} \otimes w_{j} \kappa\left(v_{i}\right),
$$

and for any $\varrho \in A^{\prime}$ we have

$$
\left(w \oplus v^{c}\right)_{\varrho}=\sum_{i, j} n_{j} \otimes m_{i}^{T} \varrho\left(w_{j} \kappa\left(v_{i}\right)\right) .
$$

Let $a \in B(K, L)$. Then using (3.9) and (3.2) we get

$$
\left(w \oplus v^{c}\right)_{\varrho} a=\sum_{i, j} n_{j} a m_{i} \varrho\left(w_{j} k\left(v_{i}\right)\right)=(\mathrm{id} \otimes \varrho) w(a \otimes I) v^{-1} .
$$

This formula shows that $a$ is $\left(w \oplus v^{c}\right)$-invariant element if and only if $w(a \otimes I) v^{-1}$ $=a \otimes I$. The latter is equivalent to $w(a \otimes I)=(a \otimes I) v$ and Statement 1 follows.

Let $b \in B(L, K)$. Then

$$
\left(n_{j} \otimes m_{i}^{T}\right)^{T} b=m_{i} b n_{j} .
$$

Indeed for any $a \in B(L, K)^{\prime}=B(K, L)$ we have [cf. (3.9) and (3.10)]

$$
\begin{aligned}
\left\langle\left(n_{j} \otimes m_{i}^{T}\right)^{T} b, a\right\rangle & =\left\langle b,\left(n_{j} \otimes m_{i}^{T}\right) a\right\rangle=\left\langle b, n_{j} a m_{i}\right\rangle=\operatorname{Tr}\left(b n_{j} a m_{i}\right) \\
& =\operatorname{Tr}\left(m_{i} b n_{j} a\right)=\left\langle m_{i} b n_{j}, a\right\rangle
\end{aligned}
$$

and (3.16) follows. Therefore

$$
\left(w \oplus v^{c}\right)_{\varrho}{ }^{T} b=\sum_{i, j} m_{i} b n_{j} \varrho\left(w_{j} \kappa\left(v_{i}\right)\right)=(\mathrm{id} \otimes \varrho)\left(\sum_{i, j} m_{i} b n_{j} \otimes w_{j} \kappa\left(v_{i}\right)\right),
$$

and $b$ is a $\left(w \oplus v^{c}\right)$-invariant functional if and only if

$$
\sum_{i, j} m_{i} b n_{j} \otimes w_{j} \kappa\left(v_{i}\right)=b \otimes I .
$$

Applying to both sides the mapping $T \otimes$ id and remembering that $T$ is antimultiplicative, we see that the latter is equivalent to

$$
[(T \otimes \mathrm{id}) w]\left(b^{T} \otimes I\right) v^{c}=b^{T} \otimes I .
$$

Inserting in (3.2) $v^{c}$ instead of $v$ we get $\left(v^{c}\right)^{-1}=(T \otimes \mathrm{id}) v^{c c}$. Therefore (3.18) can be rewritten in the following form

$$
[(T \otimes \mathrm{id}) w]\left(b^{T} \otimes I\right)=\left(b^{T} \otimes I\right)\left[(T \otimes \mathrm{id}) v^{c c}\right],
$$

and using again the mapping $T \otimes$ id we finally see that (3.17) is equivalent to the relation $(b \otimes I) w=v^{c c}(b \otimes I)$. Statement 2 follows.

With the same techniques one can prove Statements 3 and 4 . The details can be checked by the reader. Q.E.D.

Let $v^{\prime}=\left(T \otimes \kappa^{-1}\right) v$. Repeating the proof of Proposition 3.3 one can check that $v^{\prime}$ is a smooth non-degenerate representation. Note that $v^{c}=v$. Replacing in Proposition $3.4 .3 v$ by $v^{\prime}$ and using Proposition 2.3 we get 
Theorem 3.5. Let $v$ and $w$ be irreducible representations of $G$. Assume that $v$ is smooth and non-degenerate. Then

$$
\left(\begin{array}{c}
w \text { is equi- } \\
\text { valent to } v^{c}
\end{array}\right) \Leftrightarrow\left(\begin{array}{c}
\text { There exists a non-zero } \\
(v \oplus w) \text {-invariant element }
\end{array}\right) \text {. }
$$

In the next sections we shall prove that for any smooth representation $v$ the representations complex conjugate to $v$ and contragradient to $v$ are equivalent and that the second contragradient $v^{c c}$ is equivalent to $v$ (note that the second complex conjugate $\overline{\bar{v}}=v$ ).

\section{The Haar Measure}

It is difficult to overestimate the role of the Haar measure in the theory of compact groups. The averaging over compact groups is the main tool of the representation theory and is constantly used in the applications. For this reason the theorem stating the existence and the uniqueness of the Haar measure should be considered as the central theorem in the theory of compact groups.

In our theory the algebra of continuous functions on a compact group is replaced by a (in general) non-commutative $C^{*}$-algebra $A$. Therefore instead with measures we deal with states on $A$.

In this section the existence and the uniqueness of the state invariant under left (and simultaneously right) shifts will be established for an arbitrary compact matrix pseudogroup. For obvious reason this state is called the Haar measure.

We shall prove that also in the theory of compact matrix pseudogroups the Haar measure has all the properties that are relevant for the representation theory and other applications. In particular we shall use the Haar measure to show that any smooth representation of a compact matrix pseudogroup can be decomposed into the direct sum of irreducible representations.

Let $G=(A, u)$ be a compact matrix pseudogroup. We know that $A$ is finitely generated (by matrix elements of $u$ ). Therefore $A$ is separable and the set of faithful states is not empty. All the results obtained in this section are based on the following.

Proposition 4.1. Let @ be a faithful state defined on $A$ and $h_{\varrho}$ be the Cesaro limit of the sequence $\left(\varrho^{* n}\right)_{n=1,2, \ldots}$ (cf. Proposition 2.7). Then for any $\eta \in A^{\prime}\left(A^{\prime}\right.$ is the set of all continuous linear functionals defined on $A$ ) we have

$$
h_{\varrho} * \eta=\eta * h_{\varrho}=\eta(I) h_{\varrho} .
$$

Proof. All the linear functionals entering (4.1) are continuous. We shall use notation (2.9). By virtue of Proposition 2.5 it is sufficient to show that

$$
v_{h_{\varrho} * \eta}=v_{\eta * h_{\varrho}}=\eta(I) v_{h_{\varrho}}
$$

for any representation $v$ of $G$ belonging to the class $\mathscr{R}_{G}$.

Let $K$ be the $f$-d.c. vector space on which $v$ acts and $(\cdot \mid \cdot)_{K}$ be a non-degenerate positive sesquilinear form on $K$. Then $K$ becomes a Hilbert space and $B(K)$, 
$B(K) \otimes A$, and $B(K) \otimes B(K) \otimes A$ become $C^{*}$-algebras. According to Proposition 3.2, $v$ is an invertible element of $B(K) \otimes A$. Therefore there exists a strictly positive number $c \in \mathbb{R}$ such that

$$
\begin{aligned}
& c I_{B(K) \otimes A} \leqq v^{*} v, \\
& c I_{B(K) \otimes A} \leqq v v^{*} .
\end{aligned}
$$

Let

$$
x=v-I_{B(K) \otimes A} .
$$

At first we shall prove some estimates. Let

$$
i: B(K) \otimes A \rightarrow B(K) \otimes A \otimes A
$$

be the $C^{*}$-algebra homomorphism considered in Sect. 2. We remind that $i(m \otimes a)$ $=m \otimes I \otimes a$ for any $m \in B(K)$ and $a \in A$. Therefore

$$
(\mathrm{id} \otimes \eta \otimes \mathrm{id}) i(q)=q
$$

for any $q \in B(K) \otimes A$ and any linear functional $\eta$ defined on $A$ such that $\eta(I)=1$. By virtue of (2.3)

$$
(v \otimes I) i(x)=v \oplus x, \quad(x \otimes I) i(v)=x \oplus v .
$$

Taking into account (4.3) and (4.4) we have

$$
\begin{gathered}
c i\left(x^{*} x\right) \leqq(v \oplus x)^{*}(v \oplus x), \\
c(x \otimes I)(x \otimes I)^{*} \leqq(x \oplus v)(x \oplus v)^{*} .
\end{gathered}
$$

Applying the positivity preserving map id $\otimes h_{\varrho} \otimes \varrho$ to both sides of (4.7) and using (4.6) we get

$$
c(\mathrm{id} \otimes \varrho)\left(x^{*} x\right) \leqq\left(\mathrm{id} \otimes h_{\varrho} \otimes \varrho\right)\left[(v \oplus x)^{*}(v \oplus x)\right] .
$$

Similarly, applying $\mathrm{id} \otimes \varrho \otimes h_{\varrho}$ to both sides of (4.8) we obtain

$$
c(\mathrm{id} \otimes \varrho)\left(x x^{*}\right) \leqq\left(\mathrm{id} \otimes \varrho \otimes h_{\varrho}\right)\left[(x \oplus v)(x \oplus v)^{*}\right] .
$$

We compute the right-hand side of (4.9). Remembering that $v$ is a representation, we have [cf. (4.5)]

$$
v \oplus x=v \oplus v-v \oplus I_{B(K) \otimes A}=(\mathrm{id} \otimes \Phi) v-v \otimes I .
$$

Therefore

$$
(v \oplus x)^{*}(v \oplus x)=(\mathrm{id} \otimes \Phi)\left(v^{*} v\right)-2 \operatorname{Re}\left[\left(v^{*} \otimes I\right)(v \oplus v)\right]+v^{*} v \otimes I,
$$

where $\operatorname{Re}[a]$ denotes the hermitian part of $a: \operatorname{Re}[a]=\frac{1}{2}\left(a+a^{*}\right)$. By virtue of (1.50) and (2.25) we have

$$
\begin{aligned}
\left(\mathrm{id} \otimes h_{\varrho} \otimes \varrho\right)(\mathrm{id} \otimes \Phi)\left(v^{*} v\right) & =\left(\mathrm{id} \otimes h_{\varrho} * \varrho\right)\left(v^{*} v\right) \\
& =\left(\mathrm{id} \otimes h_{\varrho}\right)\left(v^{*} v\right) .
\end{aligned}
$$

The same result we get computing the contribution coming from the last term of (4.11):

$$
\left(\mathrm{id} \otimes h_{\varrho} \otimes \varrho\right)\left(v^{*} v \otimes I\right)=\left(\mathrm{id} \otimes h_{\varrho}\right)\left(v^{*} v\right) .
$$


Moreover using the notation $(2.9)$ we have $(\mathrm{id} \otimes \mathrm{id} \otimes \varrho)(v \oplus v)=v\left(v_{\varrho} \otimes I\right)$. Therefore

$$
\left(\mathrm{id} \otimes h_{\varrho} \otimes \varrho\right)\left[\left(v^{*} \otimes I\right)(v \oplus v)\right]=\left(\mathrm{id} \otimes h_{\varrho}\right)\left(v^{*} v\right) v_{\varrho} .
$$

The above computations show that the right-hand side of (4.9) equals to

$$
2\left(\mathrm{id} \otimes h_{\varrho}\right)\left(v^{*} v\right)-2 \operatorname{Re}\left[\left(\mathrm{id} \otimes h_{\varrho}\right)\left(v^{*} v\right) v_{\varrho}\right]=2 \operatorname{Re}\left[T\left(I_{B(K)}-v_{\varrho}\right)\right],
$$

where $T=\left(\mathrm{id} \otimes h_{\varrho}\right)\left(v^{*} v\right)$. This way we proved that

$$
c(\mathrm{id} \otimes \varrho)\left(x^{*} x\right) \leqq 2 \operatorname{Re}\left[T\left(I_{B(K)}-v_{\varrho}\right)\right] .
$$

Using the same techniques one can compute the right-hand side of (4.10). Inserting the result into (4.10), we get

$$
c(\mathrm{id} \otimes \varrho)\left(x x^{*}\right) \leqq 2 \operatorname{Re}\left[\left(I_{B(K)}-v_{\varrho}\right) T^{\prime}\right],
$$

where $T^{\prime}=\left(\mathrm{id} \otimes h_{\varrho}\right)\left(v v^{*}\right)$.

By virtue of (2.10) and (2.25) we have $\left(I_{B(K)}-v_{\varrho}\right) v_{h_{\varrho}}=v_{h_{\varrho}}-v_{h_{\varrho}}=0$, and similarly $v_{h_{\varrho}}\left(I_{B(H)}-v_{\varrho}\right)=0$. Therefore multiplying both sides of $(4.12)$ by $v_{h_{\varrho}}$ from the right and by $v_{h_{e}}^{*}$ from the left (clearly this operation preserves the inequality relation) we obtain zero on the right-hand side:

$$
v_{h_{\varrho}}^{*} c(\mathrm{id} \otimes \varrho)\left(x^{*} x\right) v_{h_{\varrho}} \leqq 0 .
$$

Remembering that $\varrho$ is faithful we see that

$$
\left(v_{h_{\varrho}}^{*} \otimes I\right) x^{*} x\left(v_{h_{\varrho}} \otimes I\right)=0
$$

and

$$
x\left(v_{h_{\varrho}} \otimes I\right)=0 .
$$

Similarly multiplying both sides of (4.13) by $v_{h_{Q}}$ from the left and by $v_{h_{Q}}^{*}$ from the right and repeating the arguments used above we obtain

$$
\left(v_{h_{e}} \otimes I\right) x=0 .
$$

Replacing in (4.14) and (4.15) $x$ by the right-hand side of (4.5) and comparing the two relations, we see that

$$
\left(v_{h_{e}} \otimes I\right) v=v\left(v_{h_{e}} \otimes I\right)=v_{h_{\varrho}} \otimes I .
$$

Applying $(\mathrm{id} \otimes \eta)$ to all segments of this equation we get $v_{h_{Q}} v_{\eta}=v_{\eta} v_{h_{Q}}=\eta(I) v_{h_{Q}}$ and [cf. (2.10)] relation (4.2) follows. Q.E.D.

Let $h^{\prime}$ be a normalized (i.e. $h^{\prime}(I)=I$ ) continuous linear functional on $A$ such that for any $\eta \in A^{\prime}$, we have

$$
h^{\prime} * \eta=\eta * h^{\prime}=\eta(I) h^{\prime} .
$$

For example $h^{\prime}=h_{\varrho^{\prime}}$, where $\varrho^{\prime}$ is another faithful state on $A$. Then inserting $\eta=h^{\prime}$ in (4.1) and $\eta=h_{\varrho}$ in (4.17) and comparing the two relations we get $h^{\prime}=h_{\varrho}$. In particular $h_{\varrho^{\prime}}=h_{\varrho}$ for any faithful state $\varrho^{\prime}$ on $A$. This way we proved that for all faithful states $\varrho$ on $A$ the Cesàro limit (2.24) is the same. This common limit will be 
denoted by $h: h=h_{\varrho}$ for any faithful state $\varrho$ on $A$. Moreover we have

$$
h * \eta=\eta * h=\eta(I) h
$$

for any $\eta \in A^{\prime}$.

Let $a \in A$. Then

$$
(\eta * h)(a)=(h * \eta)(a)=\eta(I) h(a),
$$

and using (1.51) we obtain

$$
\eta(h * a)=\eta(a * h)=\eta(h(a) I)
$$

for any $\eta \in A^{\prime}$. Therefore

$$
h * a=a * h=h(a) I
$$

for any $a \in A$. If $h^{\prime}$ is a state of $A$ such that

$$
h^{\prime} * a=a * h^{\prime}=h^{\prime}(a) I
$$

for any $a \in A$, then

$$
h^{\prime}(a) I=h^{\prime}(a) I * h=h^{\prime} * a * h=h^{\prime} * h(a) I=h(a) I
$$

and $h^{\prime}=h$.

This way we proved the first three properties listed in the following theorem

Theorem 4.2. Let $G=(A, u)$ be a compact matrix pseudogroup, $\mathscr{A}$ be the *-subalgebra of A generated by matrix elements of $u$ and $\kappa: \mathscr{A} \rightarrow \mathscr{A}$ be the coinverse associated with $G$. Then there exists a state $h$ on $A$ such that

1.

$$
h(a)=\underset{n \rightarrow \infty}{C-\lim } \varrho^{* n}(a)
$$

for any faithful state $\varrho$ on $A$ and any $a \in A$.

2.

$$
\eta * h=h * \eta=\eta(I) h
$$

for any continuous functional $\eta$ defined on $A$.

3.

$$
a * h=h * a=h(a) I
$$

for any $a \in A$.

4. For any $a \in \mathscr{A}$,

$$
h(\kappa(a))=h(a) .
$$

5. For any $a \in \mathscr{A}$,

$$
\left(\begin{array}{c}
a \geqq 0 \\
h(a)=0
\end{array}\right) \Rightarrow(a=0) .
$$

Each of the property 1-3 determines the state $h$ uniquely.

Proof of Property 4. The compositions $h \circ \kappa^{-1}$ and $h \circ \kappa$ are linear functionals defined on $\mathscr{A}$ assuming value 1 at $I$. Let $a \in \mathscr{A}$. Then using (1.51) and (4.20) we have

$$
\left(h *\left(h \circ \kappa^{-1}\right)\right)(\kappa(a))=\left(h \circ \kappa^{-1}\right)(\kappa(a) * h)=\left(h \circ \kappa^{-1}\right)(h(\kappa(a)) I)=h(\kappa(a)) .
$$


On the other hand using (1.50), (1.47), (1.51), and (4.20), we compute

$$
\begin{aligned}
\left(h *\left(h \circ \kappa^{-1}\right)\right)(\kappa(a)) & =\left(h \otimes\left(h \circ \kappa^{-1}\right)\right) \Phi(\kappa(a)) \\
& =\left(h \otimes\left(h \circ \kappa^{-1}\right)\right) \sigma_{A}(\kappa \otimes \kappa) \Phi(a) \\
& =\left(\left(h \circ \kappa^{-1}\right) \otimes h\right)(\kappa \otimes \kappa) \Phi(a) \\
& =(h \otimes(h \circ \kappa)) \Phi(a)=(h *(h \circ \kappa))(a) \\
& =(h \circ \kappa)(a * h)=(h \circ \kappa)(h(a) I)=h(a),
\end{aligned}
$$

and formula (4.21) holds. Q.E.D.

The proof of the last property [implication (4.22)] will be given later.

In our theory the algebra $A$ plays the role of the algebra of all continuous functions on a compact group. Therefore the states of $A$ correspond to normalized positive measures on the group. Properties 2, 3, and 4 are characteristic for a left and right invariant Haar measure. Therefore in our theory the state $h$ plays the role of the Haar measure and in the following it will be called the Haar measure.

The proof of the existence of the Haar measure should be considered as an essential achievement of our approach. In the earlier theories $[6,11,5,13]$ the existence of the Haar measure was stated by an axiom with the great methodological disaccord with the theory of (locally) compact groups, where the proof of existence of the Haar measure is highly non-trivial.

The following statement shows how the Haar measure can be used to the investigation of invariant elements and functionals:

Proposition 4.3. Let $v$ be a representation of $G$ acting on a $f$-d.c. vector space $K$ and $E=v_{h}=(\mathrm{id} \otimes h) v$. Then

1. $E \in \operatorname{Mor}(v, v)$.

2. $E$ is a projection onto the space of all $v$-invariant elements.

3. $E^{T}$ is a projection onto the space of all v-invariant functionals.

4. For any non-zero v-invariant element $x \in K$ one can find a $v$-invariant functional $x^{\prime} \in K^{\prime}$ such that $\left\langle x^{\prime}, x\right\rangle$ is strictly positive.

Proof. By virtue of (4.19) and (2.10) we have

$$
v_{\eta} E=E v_{\eta}=\eta(I) E
$$

for any $\eta \in A^{\prime}$. The first equality shows that $E \in \operatorname{Mor}(v, v)$ (cf. Proposition 2.1). Moreover inserting $\eta=h$ we get $E^{2}=E$, i.e. $E$ is a projection. Consequently $E^{T}$ is a projection.

Assume that $x \in$ range $E$. Then $E x=x$ and the above formula shows that $v_{\eta} x$ $=\eta(I) x$ for any $\eta \in A^{\prime}$. Therefore $x$ is a $v$-invariant element. Conversely if $x \in K$ is $v$-invariant then $v_{\eta} x=\eta(I) x$ for any $\eta \in A^{\prime}$ and setting $\eta=h$ we get $E x=x$. Statement 2 is proved.

Assume that $x^{\prime} \in$ range $E^{T}$. Then $x^{\prime} \circ E=x^{\prime}$, and the above formula shows that $x^{\prime} \circ v_{\eta}=\eta(I) x^{\prime}$ for any $\eta \in A^{\prime}$. Therefore $x^{\prime}$ is a $v$-invariant functional. Conversely if $x^{\prime} \in K^{\prime}$ is $v$-invariant then $x^{\prime} \circ v_{\eta}=\eta(I) x^{\prime}$ for any $\eta \in A^{\prime}$ and setting $\eta=h$ we get $x^{\prime} \circ E=x^{\prime}$. Therefore $E^{T} x^{\prime}=x^{\prime}$. Statement 3 is proved. 
Let $x$ be a non-zero $v$-invariant element of $K$. Then there exists $y^{\prime} \in K^{\prime}$ such that $\left\langle y^{\prime}, x\right\rangle>0$. Let $x^{\prime}=E^{T} y^{\prime}$. Then $x^{\prime}$ is a $v$-invariant functional and $\left\langle x^{\prime}, x\right\rangle=\left\langle E^{T} y^{\prime}, x\right\rangle$ $\left.=\left\langle y^{\prime}, E x\right\rangle=\left\langle y^{\prime}, x\right\rangle\right\rangle 0$. Q.E.D.

In many interesting cases the space of all $v$-invariant elements is onedimensional. Then the space of all $v$-invariant functionals is also one-dimensional (always $\operatorname{dim} E K=\operatorname{dim} E^{T} K^{\prime}$ ) and choosing a non-zero $v$-invariant element $x \in K$ and a non-zero $v$-invariant functional $x^{\prime} \in K^{\prime}$, we have

$$
E y=\frac{\left\langle x^{\prime}, y\right\rangle}{\left\langle x^{\prime}, x\right\rangle} x
$$

for any $y \in K$. Indeed the operator $E$ introduced by (4.23) is the only projection such that range $E=\mathbb{C} x$ and range $E^{T}=\mathbb{C} x^{\prime}$.

We shall use the last statement of Proposition 4.3 with $v$ replaced by $w \oplus v^{c}$. According to Proposition 3.4 the set of all $\left(w \oplus v^{c}\right)$-invariant elements coincides with $\operatorname{Mor}(v, w)$ and the set of all $\left(w \oplus v^{c}\right)$-invariant functionals coincides with $\operatorname{Mor}\left(w, v^{c c}\right)$. Taking into account (3.10) we get

Proposition 4.4. Let $v$ and $w$ be finite-dimensional representations of $G$. Assume that $v$ is smooth and non-degenerate. Then for any non-zero $a \in \operatorname{Mor}(v, w)$, there exists $b \in \operatorname{Mor}\left(w, v^{c c}\right)$ such that $\operatorname{Tr} b a>0$.

Now we can prove the following fundamental

Theorem 4.5. Any finite-dimensional smooth representation of a compact matrix pseudogroup is equivalent to a direct sum of irreducible representations.

Proof. By virtue of Proposition 3.1 we may restrict our considerations to nondegenerate representations. Then the theorem follows immediately from the following.

Proposition 4.6. Let $w$ be a representation of $G$ acting on a $f$-d.c. vector space $L$, $K \subset L$ be a $w$-invariant subspace and $v$ be the restriction of $w$ to $K$. We assume that $v$ is smooth, non-degenerate and irreducible. Then $K$ has a w-invariant complement.

Proof. The embedding $a: K \rightarrow L$ belongs to $\operatorname{Mor}(v, w)$. According to Proposition 4.4 one can find $b: L \rightarrow K$ intertwining $w$ with $v^{c c}$ such that Trba>0. Let $L_{1}=\operatorname{ker} b$. Then $L_{1}$ is $w$-invariant.

Clearly $\operatorname{dim} L_{1}+\operatorname{dim} K \geqq \operatorname{dim} L$. Assume that $L \neq L_{1} \oplus K$. Then $L_{1} \cap K$ is a nonzero $v$-invariant subspace of $K$. Remembering that $v$ is irreducible we get $L_{1} \cap K=K$. Consequently $K \subset L_{1}$ and $b a=\left.b\right|_{K}=0$, which is in contradiction with Tr $b a>0$. Therefore $L=L_{1} \oplus K$ and $L_{1}$ is a $w$-invariant complement of $K$. Q.E.D.

This ends the proof of Theorem 4.5. Q.E.D.

Assume for the moment that $G$ is abelian. Then the convolution product is commutative and taking into account formula (2.10) and Proposition 2.2 one can easily show that all irreducible representations of $G$ are one-dimensional. Using Theorem 4.5 we can decompose the fundamental representation of $G$ into a direct sum of one-dimensional representations. In other words there exists an invertible 
matrix $t \in M_{N}(\mathbb{C})$ such that the matrix $u^{\prime}=\left(t^{-1} \otimes I\right) u(t \otimes I)$ is diagonal. Then $G^{\prime}=\left(A, u^{\prime}\right)$ is a compact matrix pseudogroup obtained from $G$ by a similarity transformation. Let $\gamma_{1}, \gamma_{2}, \ldots, \gamma_{N}$ be diagonal elements of $u^{\prime}$. By virtue of (1.2), (1.5), and (1.6),

$$
\begin{aligned}
& \Phi\left(\gamma_{n}\right)=\gamma_{n} \otimes \gamma_{n}, \\
& \kappa\left(\gamma_{n}\right)=\gamma_{n}^{-1}
\end{aligned}
$$

for $n=1,2, \ldots, N$. Computing the norm of both sides of (4.24) we get $\left\|\gamma_{n}\right\|^{2}=\left\|\Phi\left(\gamma_{n}\right)\right\|$ $\leqq\left\|\gamma_{n}\right\|$ and $\left\|\gamma_{n}\right\| \leqq 1$. Taking the inverses of both sides of (4.24) and using the same trick we get $\left\|\gamma_{n}^{-1}\right\| \leqq 1$. Therefore all $\gamma_{n}$ are unitary. Let $\Gamma$ be the subgroup of unitaries of $A$ generated by $\gamma_{1}, \gamma_{2}, \ldots, \gamma_{N}$. Clearly $\Phi(\gamma)=\gamma \otimes \gamma$ for any $\gamma \in \Gamma$.

Let $\pi$ be the universal representation of $A$ (i.e. the direct sum of all cyclic representations). Then any representation of $A$ is contained in a multiple of $\pi$. Let $U=\left.\pi\right|_{\Gamma}$. Then $U$ is a unitary representation of $\Gamma$ satisfying condition (1.26) [Indeed $U \otimes U=\left.(\pi \otimes \pi) \circ \Phi\right|_{\Gamma}$ and $(\pi \otimes \pi) \circ \Phi$ is contained in a multiple of $\pi$.] and $G^{\prime}$ is identical with the pseudogroup $\left(C^{*}(U), u\right)$ described in Sect. 1. This proves Theorem 1.7.

We turn back to the general case. Let $\hat{G}$ be the set of all equivalency classes of irreducible representations of $G$ belonging to $\mathscr{R}_{G}$. For any $\alpha \in \hat{G}$ we choose a representation $u^{\alpha} \in \alpha$. Let $K_{\alpha}$ be the $f$-d.c. vector space on which $u^{\alpha}$ acts, $d_{\alpha}=\operatorname{dim} K_{\alpha}$, $\left(e_{k}^{\alpha}: k=1,2, \ldots, d_{\alpha}\right)$ be a basis in $K_{\alpha}$ and $\left(m_{k l}^{\alpha} \in B\left(K_{\alpha}\right): k, l=1,2, \ldots, d_{\alpha}\right)$ be the corresponding system of matrix units: $m_{k l}^{\alpha} e_{r}^{\alpha}=\delta_{k r} e_{l}^{\alpha}\left(k, l, r=1,2, \ldots, d_{\alpha}\right)$. Then

$$
u^{\alpha}=\sum_{k l=1}^{d_{\alpha}} m_{k l}^{\alpha} \otimes u_{l k}^{\alpha}
$$

where $u_{l k}^{\alpha} \in \mathscr{A}\left(k, l=1,2, \ldots, d_{\alpha}\right)$. Using the well known algebraic properties of the system of matrix units one can easily check that formula $(\mathrm{id} \otimes \Phi)\left(u^{\alpha}\right)=u^{\alpha} \oplus u^{\alpha}$ means that

$$
\Phi\left(u_{k l}^{\alpha}\right)=\sum_{r} u_{k r}^{\alpha} \otimes u_{r l}^{\alpha} .
$$

Similarly using Proposition 3.2.3 we get

$$
\begin{aligned}
& \sum_{r} \kappa\left(u_{k r}^{\alpha}\right) u_{r l}^{\alpha}=\delta_{k l} I, \\
& \sum_{r} u_{k r}^{\alpha} \kappa\left(u_{r l}^{\alpha}\right)=\delta_{k l} I .
\end{aligned}
$$

Proposition 4.7. The system $\left(u_{k l}^{\alpha}: \alpha \in \hat{G}, k, l=1,2, \ldots, d_{\alpha}\right)$ is a basis in the vector space $\mathscr{A}$.

Proof. By virtue of Proposition 2.5 and Theorem 4.5 any element of $\mathscr{A}$ can be written as a finite linear combination of elements $u_{k l}^{\alpha}$. We have to show that these elements are linearly independent. This fact follows immediately from the following

Lemma 4.8. Let $F$ be a finite subset of $\hat{G}$ and $\lambda_{k l}^{\alpha} \in \mathbb{C}$ for $k, l=1,2, \ldots, d_{\alpha}, \alpha \in F$. Then there exists a continuous linear functional $\varrho$ on $A$ such that

$$
\varrho\left(u_{k l}^{\alpha}\right)=\lambda_{k l}^{\alpha}
$$

for all $k, l=1,2, \ldots, d_{\alpha}$ and $\alpha \in F$. 
Proof of the lemma. Let $A^{\prime}$ be the set of all continuous linear functionals defined on $A$. We shall use notation (2.9). In the algebra

$$
B=\sum_{\alpha \in F}^{\oplus} B\left(K_{\alpha}\right)
$$

we consider the subset

$$
B_{1}=\left\{\sum_{\alpha \in F}^{\oplus} u_{\varrho}^{\alpha}: \varrho \in A^{\prime}\right\}
$$

By virtue of (2.10) $B_{1}$ is a subalgebra of $B$. For any $\beta \in F$ the map

$$
B_{1} \ni \sum_{\alpha \in F}^{\oplus} u_{\varrho}^{\alpha} \mapsto u_{\varrho}^{\beta} \in B\left(K_{\beta}\right)
$$

is an irreducible representation of $B_{1}$ (a subspace invariant under all $u_{\varrho}^{\beta}$ is $u^{\beta}$-invariant). Proposition 2.1 shows that the representations (4.30) are pairwise inequivalent. Therefore using the Burnside theorem (e.g. [8]) and Theorem 2.5.A of [15] we obtain $B_{1}=B$. It means that for any sequence $\left(t_{\alpha} \in B\left(K_{\alpha}\right): \alpha \in F\right)$ there exists $\varrho \in A^{\prime}$ such that $t_{\alpha}=u_{\varrho}^{\alpha}$ for all $\alpha \in F$. Taking $t_{\alpha}=\sum m_{k l}^{\alpha} \lambda_{l k}^{\alpha}$ (summation over $\left.k, l=1,2, \ldots, d_{\alpha}\right)$ and using (4.25) we obtain (4.29). Q.E.D.

This ends the proof of Proposition 4.7. Q.E.D.

Let $a \in \mathscr{A}$. Then

$$
a=\sum_{\alpha \in F} \sum_{k l=1}^{d_{\alpha}} a_{k l}^{\alpha} u_{k l}^{\alpha}
$$

where $F$ is a finite subset of $\hat{G}, a_{k l}^{\alpha} \in \mathbb{C}\left(k, l=1,2, \ldots, d_{\alpha} ; \alpha \in F\right)$. By virtue of Lemma 4.8 one can find $\varrho_{r s}^{\beta} \in A^{\prime}\left(r, s=1,2, \ldots, d_{\beta} ; \beta \in F\right)$ such that $\varrho_{r s}^{\beta}\left(u_{k l}^{\alpha}\right)=\delta_{\beta \alpha} \delta_{r k} \delta_{s l}$. Using (1.49) and (4.26) we get

$$
a * \varrho_{r s}^{\beta}=\sum_{l=1}^{d_{\beta}} a_{r l}^{\beta} u_{s l}^{\beta},
$$

and taking into account (4.27) we obtain

$$
\sum_{s=1}^{d_{\beta}} \kappa\left(u_{k s}^{\beta}\right)\left(a * \varrho_{r s}^{\beta}\right)=a_{r k}^{\beta} I
$$

for all $r, k=1,2, \ldots, d_{\beta}$ and $\beta \in F$.

Now we can complete the proof of Theorem 4.2.

Proof of the Implication (4.22). Assume that $a$ given by (4.31) is positive and $h(a)=0$. Then for any state $\varrho$ of $A, a * \varrho$ is positive and [cf. (1.51) and (4.19)] $h(a * \varrho)$ $=(\varrho * h)(a)=h(a)=0$. It means that $(a * \varrho)^{1 / 2}$ belongs to the left ideal $J=\left\{a \in A: h\left(a^{*} a\right)=0\right\}$. Therefore $a * \varrho \in J$. Since any continuous linear functional on $A$ is a linear combination of states, the latter holds for any $\varrho \in A^{\prime}$. Formula (4.32) shows now that $a_{r k}^{\beta} I \in J$ and $a_{r k}^{\beta}=0$ for all $r, k=1,2, \ldots, d_{\beta}$ and $\beta \in F$. Therefore $a=0$. Q.E.D.

At the end of this section we consider certain mappings acting on $\mathscr{A} \otimes_{\text {alg }} \mathscr{A}$ that play an important role in the differential calculus on pseudogroups (cf. $[18,20]$ ). 
Let $r, r^{\prime}, s, s^{\prime}$ be linear transformations acting on $\mathscr{A} \otimes_{\text {alg }} \mathscr{A}$ such that for any $a, b$

$$
\begin{aligned}
r(a \otimes b) & =(a \otimes I) \Phi(b), \\
r^{\prime}(a \otimes b) & =\Phi(a)(b \otimes I), \\
s(a \otimes b) & =(I \otimes a) \Phi(b), \\
s^{\prime}(a \otimes b) & =\Phi(a)(I \otimes b) .
\end{aligned}
$$

Theorem 4.9. The linear transformations $r, r^{\prime}, s$, and $s^{\prime}$ are bijections of $\mathscr{A} \otimes_{\text {alg }} \mathscr{A}$ onto itself. The inverse transformations are given by the formulae

$$
\begin{aligned}
r^{-1}(a \otimes b) & =(a \otimes I)(\kappa \otimes \mathrm{id}) \Phi(b), \\
r^{-1}(a \otimes b) & =\left[\sigma_{A}\left(\kappa^{-1} \otimes \mathrm{id}\right) \Phi(b)\right](I \otimes a), \\
s^{-1}(a \otimes b) & =(b \otimes I) \sigma_{A}\left(\mathrm{id} \otimes \kappa^{-1}\right) \Phi(a), \\
s^{\prime-1}(a \otimes b) & =[(\mathrm{id} \otimes \kappa) \Phi(a)](I \otimes b),
\end{aligned}
$$

for any $a, b \in \mathscr{A}$. Moreover for any $a \in \mathscr{A}$

$$
r^{\prime}\left(r^{-1}(I \otimes a)\right)=I \otimes \kappa(a) .
$$

Proof. We have to check that the linear mappings introduced by the formulae (4.33)-(4.36) are really the inverses of $r, r^{\prime}, s$, and $s^{\prime}$ respectively. To this end one may use formulae derived in Sect. 1. However it is simpler to use formulae (4.26)-(4.28). By virtue of Proposition 4.7 it is sufficient to perform computations for $a=u_{k l}^{\alpha}$ and $b=u_{r s}^{\beta}$ (where $\alpha, \beta \in \hat{G} ; k, l=1,2, \ldots, d_{\alpha}$ and $\left.r, s=1,2, \ldots, d_{\beta}\right)$. For example we compute

$$
\begin{aligned}
r\left(r^{-1}(a \otimes b)\right) & =\sum_{m=1}^{d_{\beta}} r\left(u_{k l}^{\alpha} \kappa\left(u_{r m}^{\beta}\right) \otimes u_{m s}^{\beta}\right) \\
& =\sum_{n m=1}^{d_{\beta}} u_{k l}^{\alpha} \kappa\left(u_{r m}^{\beta}\right) u_{m n}^{\beta} \otimes u_{n s}^{\beta}=a \otimes b .
\end{aligned}
$$

Therefore $r \circ r^{-1}=\mathrm{id}$. In the similar way one can check that $r^{-1} \circ r=\mathrm{id}, r^{\prime} \circ r^{-1}$ $=r^{\prime-1} \circ r^{\prime}=\mathrm{id}, \quad s \circ s^{-1}=s^{-1} \circ s=\mathrm{id}$, and $s^{\prime} \circ s^{\prime-1}=s^{\prime-1} \circ s^{\prime}=\mathrm{id} \quad$ (where $\mathrm{id}=\mathrm{id}_{\mathscr{A} \otimes_{\text {alg }} \mathscr{A}}$. Finally we have [cf. (1.47)]:

$$
\begin{aligned}
r^{\prime}\left(r^{-1}(I \otimes a)\right) & =\sum_{m=1}^{d_{\alpha}} r^{\prime}\left(\kappa\left(u_{k m}^{\alpha}\right) \otimes u_{m l}^{\alpha}\right) \\
& =\sum_{m=1}^{d_{\alpha}} \Phi\left(\kappa\left(u_{k m}^{\alpha}\right)\right)\left(u_{m l}^{\alpha} \otimes I\right) \\
& =\sum_{n m=1}^{d_{\alpha}}\left(\kappa\left(u_{n m}^{\alpha}\right) \otimes \kappa\left(u_{k n}^{\alpha}\right)\right)\left(u_{m l}^{\alpha} \otimes I\right) \\
& =I \otimes \kappa\left(u_{k l}^{\alpha}\right)=I \otimes \kappa(a) . \quad \text { Q.E.D. }
\end{aligned}
$$




\section{Unitary Representations, Characters, and Modular Properties}

In the theory of representations of compact groups an important role is played by unitary representations. There is a theorem saying that any finite-dimensional representation is equivalent to a unitary one. Analogous results can be obtained in our theory. As in the previous sections $G=(A, u)$ is a compact matrix pseudogroup, $\Phi$ and $\kappa$ are comultiplication and coinverse associated with $G$ and $\mathscr{A}$ is the dense *-subalgebra generated by matrix elements of $u$. We start with the following definition:

Let $K$ be a finite dimensional Hilbert space. Then $B(K)$ and $B(K) \otimes A$ are $C^{*}$-algebras. We say that a representation $v$ of $G$ acting on $K$ is unitary if $v$ is a unitary element of $B(K) \otimes A$.

One can easily check that the direct sum, the tensor product and the complex conjugation applied to the unitary representations produce a unitary representation. Moreover if $v$ and $w$ are unitary representations acting on a $\mathrm{f}-\mathrm{d}$. Hilbert spaces $K$ and $L$ respectively and $a \in \operatorname{Mor}(v, w)$, then $a^{*} \in \operatorname{Mor}(w, v)$. Indeed if $(a \otimes I) v=w(a \otimes I)$, then $v^{*}\left(a^{*} \otimes I\right)=\left(a^{*} \otimes I\right) w^{*}$ and multiplying both sides from the left by $v$ and from the right by $w$ we get $\left(a^{*} \otimes I\right) w=v\left(a^{*} \otimes I\right)$.

It is not easy to show that any subrepresentation of a unitary representation $w$ is unitary and that the orthogonal complement of a $w$-invariant subspace is $w$-invariant. In order to prove these facts one has to use in an essential way the results contained in Sect. 4. At this moment we shall prove only the following simple statement:

Proposition 5.1. Let w be a unitary representation of $G$ acting on a $\mathrm{f}-\mathrm{d}$. Hilbert space $L, v$ be a subrepresentation of $w$ acting on a $w$-invariant subspace $K \subset L$ and $K^{\perp}$ be the orthogonal complement of $K$. Assume that $v$ is an invertible element of $B(K) \otimes A$. Then $v$ is unitary; $K^{\perp}$ is $w$-invariant and $w_{K^{\perp}}$ is unitary.

Proof. Let $P$ be the orthogonal projection onto $K$. Then the restriction of $w$ to $K$ is given by the formula

$$
v=w(P \otimes I) .
$$

Let us notice that $P\left(P \otimes I\right.$ respectively) can be identified with $I_{B(K)}\left(I_{B(K) \otimes A}\right.$ respectively). Remembering that $w$ is unitary we have

$$
v^{*} v=(P \otimes I) w^{*} w(P \otimes I)=P \otimes I .
$$

We assumed that $v$ is invertible. (5.2) shows that the inverse of $v$ coincides with $v^{*}$, i.e. $v$ is unitary. Therefore $v v^{*}=I_{B(K) \otimes A}=P \otimes I$. Taking into account Eq. (5.1), we get $w(P \otimes I) w^{*}=P \otimes I$, and finally $w(P \otimes I)=(P \otimes I) w$. It means that $P \in \operatorname{Mor}(w, w)$. Therefore $K^{\perp}=\operatorname{ker} P$ is $w$-invariant.

Let $P^{\perp}=I_{B(L)}-P$ be the orthogonal projection onto $K^{\perp}$. Then $P^{\perp} \otimes I$ commutes with $w$ and $w_{1}=w\left(P^{\perp} \otimes I\right)$ is the restriction of $w$ to $K$. Repeating computation (5.2) we get

$$
w_{1}^{*} w_{1}=P^{\perp} \otimes I .
$$

On the other hand

$$
w_{1} w_{1}^{*}=w\left(P^{\perp} \otimes I\right) w^{*}=\left(P^{\perp} \otimes I\right) w w^{*}=P^{\perp} \otimes I .
$$

The last two formulae show that $w_{1}$ is a unitary element of $B\left(K^{\perp}\right) \otimes A$. Q.E.D. 
Let $K$ be a $f$-d. Hilbert space. According to the Fréchet-Riesz theorem one may identify $\bar{K}$ with $K^{\prime}$ in such a way that $(x \mid y)=\langle\bar{x}, y\rangle$ for any $x, y \in K$. Let $v$ be a nondegenerate smooth representation of $G$ acting on $K$. One can easily check that $v$ is unitary if and only if $v^{c}=\bar{v}$.

Theorem 5.2. Let $v$ be a representation of $G$ acting on a f-d.c. vector space K. Assume that $v$ is an invertible element of $B(K) \otimes A$. Then there exists a scalar product on $K$, providing $K$ a Hilbert space structure such that $v$ becomes a unitary representation.

Proof. It is sufficient to show that $v$ is equivalent to a unitary representation.

We endow $K$ with a strictly positive sesquilinear form $(\cdot \mid \cdot)$. Then $(K,(\cdot \mid \cdot))$ is a Hilbert space and $B(K)$ and $B(K) \otimes A$ become $C^{*}$-algebras. Since $v$ is invertible, there exists a positive constant $c$ such that $c I_{B(K) \otimes A} \leqq v^{*} v$. Therefore

$$
Q=(\mathrm{id} \otimes h)\left(v^{*} v\right)
$$

is a positive invertible operator acting on $K$. We claim that

$$
v^{*}(Q \otimes I) v=Q \otimes I .
$$

Indeed assuming that $v=\sum m_{r} \otimes v_{r}\left[\right.$ where $\left.m_{r} \in B(K), v_{r} \in A, r=1,2, \ldots, R\right]$ we have

$$
\begin{gathered}
Q=\sum_{r r^{\prime}} m_{r} * m_{r^{\prime}} h\left(v_{r} * v_{r^{\prime}}\right), \\
(\mathrm{id} \otimes \Phi) v=v \oplus v=\sum_{r, s} m_{r} m_{s} \otimes v_{r} \otimes v_{s}, \\
(\mathrm{id} \otimes \Phi)\left(v^{*} v\right)=\sum_{\substack{r r^{\prime} \\
s s^{\prime}}} m_{s} * m_{r}^{*} m_{r^{\prime}} m_{s^{\prime}} \otimes v_{r} * v_{r^{\prime}} \otimes v_{s}{ }^{*} v_{s^{\prime}} .
\end{gathered}
$$

Applying (id $\otimes h \otimes \mathrm{id})$ to the right-hand side of (5.4) we get

$$
\begin{gathered}
\sum_{s s^{\prime}} m_{s}^{*}\left(\sum_{r r^{\prime}} m_{r}^{*} m_{r^{\prime}} h\left(v_{r}^{*} v_{r^{\prime}}\right)\right) m_{s^{\prime}} \otimes v_{s}^{*} v_{s^{\prime}} \\
=\sum_{s s^{\prime}} m_{s}^{*} Q m_{s^{\prime}} \otimes v_{s}^{*} v_{s^{\prime}}=v^{*}(Q \otimes I) v .
\end{gathered}
$$

On the other hand $(h \otimes \mathrm{id}) \Phi(a)=a * h=h(a) I$. Therefore applying $(\mathrm{id} \otimes h \otimes \mathrm{id})$ to the left-hand side of (5.4), we get $(\mathrm{id} \otimes h)\left(v^{*} v\right) \otimes I=Q \otimes I$, and formula (5.3) follows.

Let $w=\left(Q^{1 / 2} \otimes I\right) v\left(Q^{-1 / 2} \otimes I\right)$. Then $w$ is a representation of $G$ equivalent to $v$ $\left(Q^{1 / 2} \in \operatorname{Mor}(v, w)\right), w$ is an invertible element of $B(K) \otimes A$ and formula (5.3) shows that $w^{*} w=I$. Hence $w$ is unitary. Q.E.D.

In particular for any $\alpha \in \hat{G}$, representation $u^{\alpha}$ considered in Sect. 4 can be made unitary by a suitable choice of scalar product in $K_{\alpha}$. In what follows we shall assume that the basis $\left(e_{k}^{\alpha}: k=1,2, \ldots, d_{\alpha}\right)$ is orthonormal with respect to this scalar product. Then matrix $\left(u_{k l}^{\alpha}\right)_{k, l=1,2, \ldots, d_{\alpha}}$ is unitary and using (4.27) we get

$$
\kappa\left(u_{k l}^{\alpha}\right)=u_{l k}^{\alpha *}
$$

for any $\alpha \in \hat{G}$ and $k, l=1,2, \ldots, d_{\alpha}$. The contragradient representation can be now written in the form

$$
u^{\alpha c}=\sum_{k, l} m_{l k}^{\alpha T} \otimes u_{l k}^{\alpha *}
$$


Combining Proposition 4.3.2 and 3 with Proposition 3.4.1 and 4 we immediately get

Theorem 5.3. Let $v$ and $w$ be $f$-d. representations of $G$. Assume that $v$ is smooth and non-degenerate.

1. If $\operatorname{Mor}(v, w)=\{0\}$, then

$$
(\mathrm{id} \otimes h)\left(w \oplus v^{c}\right)=0 .
$$

2. If $\operatorname{Mor}(w, v)=\{0\}$, then

$$
(\mathrm{id} \otimes h)\left(v^{c} \oplus w\right)=0 .
$$

Let $\alpha, \beta \in \hat{G}$ and $\alpha \neq \beta$. Then representations $u^{\alpha}$ and $u^{\beta}$ are not equivalent and using Proposition 2.3 we have $\operatorname{Mor}\left(u^{\alpha}, u^{\beta}\right)=\{0\}$ and $\operatorname{Mor}\left(u^{\beta}, u^{\alpha}\right)=\{0\}$. Inserting $v=u^{\alpha}$ and $w=u^{\beta}$ in (5.7) and (5.8) we obtain [cf. (2.15), (4.25), and (5.6)]

$$
\begin{aligned}
& h\left(u_{k l}^{\beta} u_{m n}^{\alpha *}\right)=0 \\
& h\left(u_{m n}^{\alpha} * u_{k l}^{\beta}\right)=0,
\end{aligned}
$$

for any $k, l=1,2, \ldots, d_{\beta}$ and $m, n=1,2, \ldots, d_{\alpha}$.

In order to derive the corresponding formulae for $\beta=\alpha$ we have to prove a result corresponding to Theorem 5.3. In the formulation of the following theorem we use the identifications described in Sect. 3.

Theorem 5.4. Let $v$ be a smooth non-degenerate irreducible representation of $G$ acting on a $f$-d.c. vector space $K$. Then there exists one and only one invertible operator $F \in B(K)$ such that $\operatorname{Tr} F=\operatorname{Tr} F^{-1}>0$ and

1.

$$
\operatorname{Mor}\left(v, v^{c c}\right)=\{\lambda F: \lambda \in \mathbb{C}\} \text {. }
$$

2. For any $a \in B(K)=K \otimes K^{\prime}$,

$$
(\mathrm{id} \otimes h)\left(v \oplus v^{c}\right) a=\frac{\operatorname{Tr}(F a)}{\operatorname{Tr} F} I_{B(K)} .
$$

3. For any $b \in B\left(K^{\prime}\right)=K^{\prime} \otimes K$,

$$
(\mathrm{id} \otimes h)\left(v^{c} \oplus v\right) b=\frac{\operatorname{Tr} b}{\operatorname{Tr}\left(F^{-1}\right)}\left(F^{-1}\right)^{T} .
$$

If $K$ is a Hilbert space and $v$ is unitary, then $F$ is a strictly positive element of the $C^{*}$-algebra $B(K)$.

Proof. According to Proposition 4.4 (with $w=v$ and $a=I_{B(K)}$ ) there exists $F \in \operatorname{Mor}\left(v, v^{c c}\right)$ such that $\operatorname{Tr} F>0$. Invertibility of $F$ and (5.11) follows directly from Proposition 2.3.

By virtue of Proposition 2.2 and Proposition 3.4.1, $I_{B(K)}$ is the only up to a numerical factor $\left(v \oplus v^{c}\right)$-invariant element. Similarly (5.11) and Proposition 3.4.2 show that $F$ is the only up to a numerical factor $\left(v \oplus v^{c}\right)$-invariant functional. Now formula (5.12) follows from Proposition 4.3 applied to the representation $v \oplus v^{c}$. Indeed inserting in (4.23) $I_{B(K)}, F$ and a instead of $x, x^{\prime}$ and $y$ respectively and taking into account (3.10) we obtain (5.12). 
We know that $F \in \operatorname{Mor}\left(v, v^{c c}\right)$. Consequently $F^{-1} \in \operatorname{Mor}\left(v^{c c}, v\right)$, and using Proposition 2.3 and Proposition 3.4.3 we see that $\left(F^{-1}\right)^{T}$ is the only up to a numerical factor $\left(v^{c} \oplus v\right)$-invariant element. Similarly Proposition 2.2 and Proposition 3.4.4 show that $I_{B(K)}$ is the only up to a numerical factor $\left(v^{c} \oplus v\right)$-invariant functional. Formula (4.23) can be applied again. Replacing $x, x^{\prime}$ and $y$ by $\left(F^{-1}\right)^{T}$, $I_{B(K)}$ and $b$ respectively and taking into account (3.10) we obtain (5.13).

By virtue of Proposition 5.2 we may assume that $K$ is a Hilbert space and that $v$ is unitary. Then replacing in (3.15) $v^{-1}$ by $v^{*}$ we see that (5.12) is positive for any positive $a \in B(K)$. Therefore $F>0, F^{-1}>0$, and $\operatorname{Tr}\left(F^{-1}\right)>0$. Up to this moment $F$ was defined uniquely up to a positive factor. We use this freedom in order to have $\operatorname{Tr}\left(F^{-1}\right)=\operatorname{Tr} F$. Clearly this condition fixes $F$ completely. Q.E.D.

For any $\alpha \in \hat{G}$ the operator $F$ related to representation $u^{\alpha}$ will be denoted by $F_{\alpha}$. Inserting in (5.12) and (5.13) $u^{\alpha}$ given by (4.25), $u^{\alpha c}$ given by (5.6), $a=m_{l k}^{\alpha}$ and $b=\left(m_{m n}^{\alpha}\right)^{T}$ and performing the necessary computation, we obtain for any $\alpha \in \widehat{G}$ and $k, l, m, n=1,2, \ldots, d_{\alpha}$ the following relations

$$
\begin{aligned}
& h\left(u_{m k}^{\alpha} u_{n l}^{\alpha}\right)=\frac{1}{M_{\alpha}} \delta_{m n} \operatorname{Tr}\left(F_{\alpha} m_{l k}^{\alpha}\right), \\
& h\left(u_{k m}^{\alpha} u_{l n}^{\alpha}\right)=\frac{1}{M_{\alpha}} \delta_{m n} \operatorname{Tr}\left(F_{\alpha}{ }^{-1} m_{l k}^{\alpha}\right),
\end{aligned}
$$

where $M_{\alpha}=\operatorname{Tr} F_{\alpha}=\operatorname{Tr}\left(F_{\alpha}^{-1}\right)$.

The Haar measure need not to be central. We are going to describe the modular properties of the Haar measure. In this description we use a certain class of holomorphic functions. We say that an entire function $f$ is of exponential growth on the right half-plane if there exist constants $M>0$ and $\tau$ real such that $|f(z)|$ $<M \exp (\tau \operatorname{Re} z)$ for any $z$ in the right half-plane. The following lemma is well known (cf. [16, p. 228]).

Lemma 5.5. Let $f_{1}$ and $f_{2}$ be entire functions of exponential growth on the right halfplane. Assume that $f_{1}(z)=f_{2}(z)$ for $z=2,4,6, \ldots$. Then $f_{1}(z)=f_{2}(z)$ for all $z \in \mathbb{C}$.

Now we can formulate the main result of this section:

Theorem 5.6. There exists one and only one family $\left(f_{z}\right)_{z \in \mathbb{C}}$ of linear multiplicative functionals defined on $\mathscr{A}$ such that

1. For any $a \in \mathscr{A}$, the mapping

$$
\mathbb{C} \ni z \mapsto f_{z}(a) \in \mathbb{C}
$$

is an entire function of exponential growth on the right half-plane

2. $f_{z}(I)=1$ for all $z \in \mathbb{C}$.

3. $f_{z} * f_{z^{\prime}}=f_{z+z^{\prime}}$

for any $z, z^{\prime} \in \mathbb{C}$. Moreover $f_{0}=e$.

4. For any $z \in \mathbb{C}$ and any $a \in \mathscr{A}$,

$$
\begin{aligned}
f_{z}(\kappa(a)) & =f_{-z}(a), \\
f_{z}\left(a^{*}\right) & =\overline{f_{-\bar{z}}(a)} .
\end{aligned}
$$

In particular for purely imaginary $z, f_{z}$ is a ${ }^{*}$-character defined on $\mathscr{A}$. 
5. For any $a \in \mathscr{A}$,

$$
\kappa^{2}(a)=f_{-1} * a * f_{1} .
$$

6. For any $a \in \mathscr{A}$ and $b \in A$,

$$
h(a b)=h\left(b\left(f_{1} * a * f_{1}\right)\right) .
$$

Proof. We know that the operators $F_{\alpha}$ are positive. For any $z$ we set $F_{\alpha}^{z}=\exp \left(z \log F_{\alpha}\right)$. By virtue of Proposition 4.7 there exists a linear functional

$$
f_{z}: \mathscr{A} \rightarrow \mathbb{C},
$$

such that

$$
f_{z}\left(u_{k l}^{\alpha}\right)=\operatorname{Tr}\left(F_{\alpha}^{z} m_{k l}^{\alpha}\right)
$$

for any $\alpha \in \hat{G}$ and $k, l=1,2, \ldots, d_{x}$. We shall prove that the family of functionals $\left(f_{z}\right)_{z \in \mathbb{C}}$ introduced by (5.22) satisfies all the requirements listed in Theorem 5.6.

Statement 1 follows immediately from (5.22) and Proposition 4.7. To prove Statement 2 it is sufficient to insert in (5.22) $\alpha=$ the class of trivial representation.

Comparing (5.22) with (4.25) we see that

$$
\left(\mathrm{id} \otimes f_{z}\right) u^{\alpha}=F_{\alpha}^{z}
$$

for any $\alpha \in \hat{G}$. Clearly (5.23) is equivalent to (5.22) and defines functionals $f_{z}$ uniquely. Inserting in (5.23) $z=0$ and using Proposition 3.2.2 we get $f_{0}=e$. Moreover by virtue of (2.10),

$$
\left[i d \otimes\left(f_{z} * f_{z^{\prime}}\right)\right] u^{\alpha}=\left(\mathrm{id} \otimes f_{z}\right) u^{\alpha}\left(\mathrm{id} \otimes f_{z^{\prime}}\right) u^{\alpha}=F_{\alpha}^{z} F_{\alpha}^{z^{\prime}}=F_{\alpha}^{z+z^{\prime}}=\left(\mathrm{id} \otimes f_{z+z^{\prime}}\right) u^{\alpha}
$$

and (5.16) follows. This way Statement 3 is proved.

According to $(5.22)\left(f_{z}\left(u_{k l}^{\alpha}\right)\right)_{k, l=1,2, \ldots, d_{\alpha}}$ is the matrix representing $F_{\alpha}^{z}$ in the basis $\left(e_{k}^{\alpha}: k=1,2, \ldots, d_{\alpha}\right)$. We know that $F_{\alpha}^{z} F_{\alpha}^{-z}=I_{B\left(K_{\alpha}\right)}$. We shall use the corresponding matrix equation

$$
\sum_{r} f_{z}\left(u_{m r}^{\alpha}\right) f_{-z}\left(u_{r n}^{\alpha}\right)=\delta_{m n}
$$

Taking into account (5.22) we may rewrite relations (5.14) and (5.15) in the following way

$$
\begin{aligned}
& h\left(u_{m k}^{\alpha} u_{n l}^{\alpha *}\right)=\frac{1}{M_{\alpha}} \delta_{m n} f_{1}\left(u_{l k}^{\alpha}\right), \\
& h\left(u_{k m}^{\alpha} * u_{l n}^{\alpha}\right)=\frac{1}{M_{\alpha}} \delta_{m n} f_{-1}\left(u_{l k}^{\alpha}\right) .
\end{aligned}
$$

We shall prove Statement 6. Let

$$
a=u_{m k}^{\alpha} \quad \text { and } \quad b=u_{n l}^{\beta *},
$$

where $\alpha, \beta \in \hat{G} ; m, k=1,2, \ldots, d_{\alpha}$ and $n, l=1,2, \ldots, d_{\beta}$. Then [cf. (4.26)]

$$
f_{1} * a * f_{1}=\sum_{r, s} f_{1}\left(u_{m r}^{\alpha}\right) u_{r s}^{\alpha} f_{1}\left(u_{s k}^{\alpha}\right)
$$


By virtue of (5.9) and (5.10) both sides of (5.20) vanish for $\beta \neq \alpha$. Therefore it is sufficient to consider the case $\beta=\alpha$. Using (5.26) and (5.24) with $z=1$ we compute

$$
\begin{aligned}
h\left(b\left(f_{1} * a * f_{1}\right)\right) & =\sum_{r, s} f_{1}\left(u_{m r}^{\alpha}\right) h\left(u_{n l}^{\alpha} * u_{r s}^{\alpha}\right) f_{1}\left(u_{s k}^{\alpha}\right) \\
& =\frac{1}{M_{\alpha}} \sum_{r, s} f_{1}\left(u_{m r}^{\alpha}\right) f_{-1}\left(u_{r n}^{\alpha}\right) \delta_{l s} f_{1}\left(u_{s k}^{\alpha}\right) \\
& =\frac{1}{M_{\alpha}} \delta_{m n} f_{1}\left(u_{l k}^{\alpha}\right)=h(a b) .
\end{aligned}
$$

In the last step we used (5.25). This way we proved (5.20) for $a$ and $b$ given by (5.27). By linearity (5.20) is satisfied for any $a, b \in \mathscr{A}$ and remembering that $\mathscr{A}$ is dense in $A$ we get (5.20) in full generality. Statement 6 is proved.

It follows easily from (5.20) and (4.22) that the mapping

$$
\mathscr{A} \ni a \mapsto f_{1} * a * f_{1} \in \mathscr{A}
$$

is multiplicative. By iteration the mappings

$$
\mathscr{A} \ni a \mapsto f_{z} * a * f_{z} \in \mathscr{A}
$$

are multiplicative for $z=1,2, \ldots$ Using (1.51), (1.53), and (5.16) we get

$$
e\left(f_{z} * a * f_{z}\right)=f_{2 z}(a) \text {. }
$$

Remembering that $e$ is multiplicative we see that

$$
f_{z}(a b)=f_{z}(a) f_{z}(b)
$$

for any $a, b \in \mathscr{A}$ and $z=2,4,6, \ldots$ By virtue of Lemma 5.5(5.29) holds for any $z \in \mathbb{C}$, i.e. all functionals (5.21) are multiplicative.

Applying $f_{z}$ to both sides of (4.28) and comparing with (5.24) we get $f_{z}\left(\kappa\left(u_{r n}^{\alpha}\right)\right)$ $=f_{-z}\left(u_{r n}^{\alpha}\right)$ for any $\alpha \in \hat{G}$ and $r, n=1,2, \ldots, d_{\alpha}$. Formula (5.17) follows.

We know that $F_{\alpha}$ is positive selfadjoint. Therefore $F_{\alpha}^{-z}=\left(F_{\alpha}^{-\bar{z}}\right)^{*}$. Writing the corresponding matrix equation we get $f_{-z}\left(u_{k l}^{\alpha}\right)=\overline{f_{-\bar{z}}\left(u_{l k}^{\alpha}\right)}$. Now using (5.5) and (5.17) we have

$$
f_{z}\left(u_{l k}^{\alpha} *\right)=f_{z}\left(\kappa\left(u_{k l}^{\alpha}\right)\right)=f_{-z}\left(u_{k l}^{\alpha}\right)=\overline{f_{-\bar{z}}\left(u_{l k}^{\alpha}\right)}
$$

and (5.18) follows. Statement 4 is proved.

We know that $F_{\alpha} \in \operatorname{Mor}\left(u^{\alpha}, u^{\alpha c c}\right)$. By virtue of (3.11) the second contragradient $u^{\alpha c c}=\left(\mathrm{id} \otimes \kappa^{2}\right) u^{\alpha}$. Therefore

$$
\left(\mathrm{id} \otimes \kappa^{2}\right) u^{\alpha}=\left(F_{\alpha} \otimes I\right) u^{\alpha}\left(F_{\alpha}^{-1} \otimes I\right) .
$$

Writing the corresponding matrix equation we get

$$
\kappa^{2}\left(u_{k l}^{\alpha}\right)=\sum_{r, s} f_{1}\left(u_{k r}^{\alpha}\right) u_{r s}^{\alpha} f_{-1}\left(u_{s l}^{\alpha}\right) .
$$

On the other hand using (4.26) one can easily check that

$$
f_{-1} * u_{k l}^{\alpha} * f_{1}=\sum_{r, s} f_{1}\left(u_{k r}^{\alpha}\right) u_{r s}^{\alpha} f_{-1}\left(u_{s l}^{\alpha}\right) \text {. }
$$


This proves (5.19) for $a=u_{k l}^{\alpha}\left(\alpha \in \hat{G}, k, l=1,2, \ldots, d_{\alpha}\right)$ and (cf. Proposition 4.7) Statement 5 follows.

Let $\left(f_{z}^{\prime}\right)_{z \in \mathbb{C}}$ be another family of linear functionals on $\mathscr{A}$ such that Statements 1 , 3 , and 6 (with $f_{z}$ replaced by $f_{z}^{\prime}$ ) are satisfied. Then using (5.20) and the corresponding formula for $f_{z}^{\prime}$ and taking into account implication (4.22) one can easily check that $f_{1}^{\prime} * a * f_{1}=f_{1}^{\prime} * a * f_{1}^{\prime}$ for any $a \in \mathscr{A}$. Iterating we get $f_{z} * a * f_{z}$ $=f_{z}^{\prime} * a * f_{z}^{\prime}$ and [cf. (5.28)] $f_{2 z}(a)=f_{2 z}^{\prime}(a)$ for any $a \in \mathscr{A}$ and $z=1,2, \ldots$. Now Lemma 5.5 shows that $f_{z}=f_{z}^{\prime}$ for any $z \in \mathbb{C}$. Q.E.D.

The Haar measure need not to be faithful. The implication (4.22) means only that the intersection of $\mathscr{A}$ with the left ideal

$$
J=\left(b \in A: h\left(b^{*} b\right)=0\right\}
$$

is trivial. However using the last statement of Theorem 5.6 one can easily show that $J$ is a two-sided ideal. Let $\pi$ be the canonical projection of $A$ onto $A / J$ and $u_{r}=(\mathrm{id} \otimes \pi) u$. Then $G_{r}=\left(A / J, u_{r}\right)$ is a compact matrix pseudogroup and the Haar measure on $G_{r}$ is faithful.

For the reader's convenience and for future reference we collect all important properties of the basis $\left(u_{k l}^{\alpha}: a \in \hat{G}, k, l=1,2, \ldots, d_{\alpha}\right)$

Theorem 5.7. Let $\left\{u^{\alpha}\right\}_{\alpha \in \hat{G}}$ be the complete family of mutually non-equivalent irreducible unitary representations of $G$ belonging to the class $\mathscr{R}_{G}$ and $u_{k l}^{\alpha}$ (where $k, l=1,2, \ldots, d_{\alpha}, d_{\alpha}=\operatorname{dim} K_{\alpha}, K_{\alpha}$ is the carrier Hilbert space of $\left.u^{\alpha}, \alpha \in \hat{G}\right)$ be the matrix elements of $u^{\alpha}$ with respect to an orthonormal basis in $K_{\alpha}$. Then

1. $\left(u_{k l}^{\alpha}: \alpha \in \hat{G}, k, l=1,2, \ldots, d_{\alpha}\right)$ is a basis in the vector space $\mathscr{A}$.

2. For any $\alpha \in \hat{G}$ the matrix $\left(u_{k l}^{\alpha}\right)_{k l=1,2, \ldots, d_{\alpha}}$ is unitary.

3. For any $\alpha \in \hat{G}$ and $k, l=1,2, \ldots, d_{\alpha}$,

$$
\begin{aligned}
& \Phi\left(u_{k l}^{\alpha}\right)=\sum_{r=1}^{d_{\alpha}} u_{k r}^{\alpha} \otimes u_{r l}^{\alpha}, \\
& \kappa\left(u_{k l}^{\alpha}\right)=u_{l k}^{\alpha *} .
\end{aligned}
$$

4. For any $\alpha, \beta \in \hat{G} ; m, k=1,2, \ldots, d_{\alpha}$ and $n, l=1,2, \ldots, d_{\beta}$,

$$
\begin{aligned}
& h\left(u_{m k}^{\alpha} u_{n l}^{\beta *}\right)=\frac{1}{M_{\alpha}} \delta_{\alpha \beta} \delta_{m n} f_{1}\left(u_{l k}^{\alpha}\right), \\
& h\left(u_{k m}^{\alpha} * u_{l n}^{\beta}\right)=\frac{1}{M_{\alpha}} \delta_{\alpha \beta} \delta_{m n} f_{-1}\left(u_{l k}^{\alpha}\right),
\end{aligned}
$$

where $M_{\alpha}=f_{1}\left(\sum_{k} u_{k k}^{\alpha}\right)=f_{-1}\left(\sum_{k} u_{k k}^{\alpha}\right)$.

For any $\alpha \in \hat{G}$ we set

$$
\chi_{\alpha}=\sum_{k=1}^{d_{\alpha}} u_{k k}^{\alpha}
$$

Then $M_{\alpha}=f_{1}\left(\chi_{\alpha}\right)=f_{-1}\left(\chi_{\alpha}\right) \cdot \chi_{\alpha}$ is said to be the character of $u^{\alpha}$. Let us notice that

$$
\Phi\left(\chi_{\alpha}\right)=\sum_{k, l} u_{k l}^{\alpha} \otimes u_{l k}^{\alpha} \in A \otimes_{\mathrm{sym}} A .
$$


This fact corresponds to the well known property of characters in the standard group representation theory saying that they are invariant under inner automorphisms. (5.31) implies that

$$
\varrho * \chi_{\alpha}=\chi_{\alpha} * \varrho
$$

for any $\varrho \in A^{\prime}$. Using Theorem 5.7 and Theorem 5.6 one can easily verify the following relations:

$$
\begin{gathered}
h\left(\chi_{\alpha} \chi_{\beta}{ }^{*}\right)=h\left(\chi_{\alpha}^{*} \chi_{\beta}\right)=\delta_{\alpha \beta}, \\
h\left(\left(f_{-1} * \chi_{\alpha}\right) u_{n l}^{\beta *}\right)=h\left(u_{n l}^{\beta *}\left(f_{1} * \chi_{\alpha}\right)\right)=\frac{1}{M_{\alpha}} \delta_{\alpha \beta} \delta_{n l}, \\
h\left(\left(f_{1} * \chi_{\alpha}\right)^{*} u_{n l}^{\beta}\right)=h\left(u_{n l}^{\beta}\left(f_{-1} * \chi_{\alpha}\right)^{*}\right)=\frac{1}{M_{\alpha}} \delta_{\alpha \beta} \delta_{n l},
\end{gathered}
$$

for any $\alpha, \beta \in \hat{G}$ and $n, l=1,2, \ldots, d_{\beta}$.

Let $\varrho_{\alpha}=M_{\alpha} h\left(f_{1} * \chi_{\alpha}\right) *$ be the continuous linear functional on $A$ such that

$$
\varrho_{\alpha}(a)=M_{\alpha} h\left(\left(f_{1} * \chi_{\alpha}\right) * a\right)
$$

for any $a \in A$ (cf. [3]). Then formula (5.35) shows that

$$
\left(\mathrm{id} \otimes \varrho_{\alpha}\right) u^{\beta}=\delta_{\alpha \beta} I_{B\left(K_{\beta}\right)} .
$$

In general the character $\chi_{v}$ of any finite-dimensional representation $v$ of $G$ is introduced by the formula

$$
\chi_{v}=(\operatorname{Tr} \otimes \mathrm{id}) v .
$$

One can easily check that the characters of equivalent representations are equal. Moreover the character of the direct sum of two representations is the sum of the corresponding characters. Similarly the character of the tensor product of two representations is the product of the corresponding characters (taken in the same order). The character of the complex conjugate representation is the hermitian conjugation of the character of the original representation.

At the end we would like to show that the class of representations $\mathscr{R}_{G}$ that we mainly dealt with is rich. In particular it contains all finite-dimensional unitary representations. This fact follows immediately from

Theorem 5.8. Let $w$ be a unitary representation of $G$ acting on a $f$-d. Hilbert space $L$. Then $L$ is the orthogonal direct sum

$$
L=\sum_{t \in T}^{\oplus} K_{t}
$$

of $w$-invariant subspaces such that for any $t$ the restriction of $w$ to $K_{t}$ is equivalent to $u^{\alpha}$ for some $\alpha \in \hat{G}$. Let $T_{\alpha}$ be the set of all $t \in T$ such that $\left.w\right|_{K_{t}}$ is equivalent to $u^{\alpha}$ and $n_{\alpha}$ be the number of elements of $T_{\alpha}(\alpha \in \hat{G})$. Then

$$
\begin{aligned}
& \chi_{w}=\sum_{\alpha} n_{\alpha} \chi_{\alpha}, \\
& n_{\alpha}=h\left(\chi_{\alpha}^{*} \chi_{w}\right) .
\end{aligned}
$$

Moreover for any $\alpha \in \hat{G},\left(\mathrm{id} \otimes \varrho_{\alpha}\right) w$ is the orthogonal projection onto $\sum_{t \in T_{\alpha}}^{\oplus} K_{t}$. 
Proof. Assume that for some $\alpha \in \hat{G}$ there exists a non-zero operator intertwining $u^{\alpha}$ with $w$. Then $w$ has a subrepresentation equivalent to $u^{\alpha}$. By virtue of Proposition 5.1 we have orthogonal decomposition $L=K_{1} \oplus L_{1}$, where $K_{1}$ and $L_{1}$ are $w$-invariant, $\left.w\right|_{K_{1}}$ is equivalent to $u^{\alpha}$ and $\left.w\right|_{L_{1}}$ is unitary. Repeating this procedure as many times as possible we arrive at the orthogonal decomposition

$$
L=\sum_{t \in T}^{\oplus} K_{t} \oplus^{\prime} L,
$$

where $K_{t}(t \in T)$ and ' $L$ are $w$-invariant subspaces, for any $t \in T$ the representation $\left.w\right|_{K_{t}}$ is equivalent to $u^{\alpha}$ for some $\alpha \in \hat{G}$ and $v=\left.w\right|_{L}$ is a unitary representation such that $\operatorname{Mor}\left(u^{\alpha}, v\right)=\{0\}$ for any $\alpha \in \widehat{G}$.

Using Theorem 5.3.1 we get

$$
(\mathrm{id} \otimes h)\left(v \oplus u^{\alpha c}\right)=0
$$

for any $\alpha \in \hat{G}$. Let

$$
v=\sum_{r} m_{r} \otimes v_{r}
$$

where $m_{1}, m_{2}, \ldots, m_{R}$ are linearly independent elements of $B\left({ }^{\prime} L\right)$ and $v_{r} \in A$ $(r=1,2, \ldots, R)$. Relation $(5.42)$ means that $h\left(v_{r} u_{k l}^{\alpha}\right)=0$ for any $\alpha \in \widehat{G} ; k, l=1,2, \ldots, d_{\alpha}$ and $r=1,2, \ldots, R$. Taking into account Proposition 4.7 and remembering that $\mathscr{A}$ is dense in $A$ we see that $h\left(v_{r} a^{*}\right)=0$ for any $a \in A$. In particular $h\left(v_{r} v_{r^{\prime}}{ }^{*}\right)=0$ for any $r, r^{\prime}$ $=1,2, \ldots, R$. Now we have

$$
(\mathrm{id} \otimes h) v v^{*}=\sum m_{r} m_{r^{*}} * h\left(v_{r} v_{r^{*}} *\right)=0 .
$$

On the other hand $v v^{*}=I_{B\left(^{\prime} L\right)} \otimes I$ and $(\mathrm{id} \otimes h) v v^{*}=I_{B\left(^{\prime} L\right)}$. This contradiction shows that $\operatorname{dim}^{\prime} L=0$ and (5.41) reduces to (5.38).

Remembering that the character of the direct sum of representations is the sum of characters we get (5.39). Formula (5.40) follows immediately from (5.39) [cf. (5.33)]. The last remark is implied by (5.37). Q.E.D.

Corollary 5.9. Two unitary representations having the same character are equivalent.

Using (5.39) and (5.33) one can easily check that

$$
h\left(\chi_{w}^{*} \chi_{w}\right)=\sum_{t \in T} n_{t}^{2} .
$$

Corollary 5.10. A unitary representation $w$ is irreducible if and only if its character $\chi_{w}$ satisfies the relation $h\left(\chi_{w}^{*} \chi_{w}\right)=1$.

Let

$$
\begin{aligned}
& A_{\text {central }}=\Phi^{-1}\left(A \otimes_{\mathrm{Sym}} A\right), \\
& \mathscr{A}_{\text {central }}=A_{\text {central }} \cap \mathscr{A} .
\end{aligned}
$$

Using Proposition 4.7 and formula (4.26) one can easily check that $a \in \mathscr{A}_{\text {central }}$ if and only if $a$ is a finite linear combination of characters $\chi_{\alpha}(\alpha \in \hat{G})$. Taking into account Corollary 5.9 and remembering that the character of the tensor product of representations is the product of the corresponding characters, we get 
Proposition 5.11. The algebra $\mathscr{A}_{\text {central }}$ is commutative if and only if for any two unitary representations $v$ and $w$ of $G, v \oplus w$ is equivalent to $w \oplus v$.

Let us notice that this is the case for $G=S_{\mu} U(2)$ and $G=S_{\mu} U(3)$.

Clearly $A_{\text {central }}$ is a $C^{*}$-subalgebra of $A$. Elements of $A_{\text {central }}$ are called central.

Let us end this paper with the following question:

Is $\mathscr{A}_{\text {central }}$ dense in $A_{\text {central }}$ ?

If $G$ is a group then the answer is positive.

\section{Appendices}

\section{A 1. Haar Measure on $S_{\mu} U(2)$}

In [18] we presented the formula describing the Haar measure on the twisted $S U(2)$ group. Here we shall prove this formula.

In this Appendix $G=S_{\mu} U(2)$, where $|\mu|<1, \mu \neq 0$. Consequently $A$ is the $C^{*}$-algebra generated by two elements $\alpha$ and $\gamma$ satisfying the relations (1.33). The comultiplication and the coinverse act on generators of $A$ in the following way (cf. [18]):

$$
\begin{gathered}
\Phi(\alpha)=\alpha \otimes \alpha-\mu \gamma^{*} \otimes \gamma, \\
\Phi(\gamma)=\gamma \otimes \alpha+\alpha^{*} \otimes \gamma, \\
\Phi\left(\alpha^{*}\right)=\alpha^{*} \otimes \alpha^{*}-\mu \gamma \otimes \gamma^{*}, \\
\Phi\left(\gamma^{*}\right)=\gamma^{*} \otimes \alpha^{*}+\alpha \otimes \gamma^{*}, \\
\kappa(\alpha)=\alpha^{*}, \quad \kappa\left(\gamma^{*}\right)=-\mu^{-1} \gamma^{*}, \\
\kappa(\gamma)=-\mu \gamma, \quad \kappa\left(\alpha^{*}\right)=\alpha .
\end{gathered}
$$

The fundamental representation $u$ and its second contragradient representation $u^{c c}$ are given by

$$
u=\left(\begin{array}{cc}
\alpha, & -\mu \gamma^{*} \\
\gamma, & \alpha^{*}
\end{array}\right), \quad u^{c c}=\left(\begin{array}{cc}
\alpha, & -\mu^{-1} \gamma^{*} \\
\mu^{2} \gamma, & \alpha^{*}
\end{array}\right) .
$$

The first formula coincides with (1.34), the second can be immediately checked by direct computation using (A 1.2) (remember that $\left.u^{c c}=\left(\mathrm{id} \otimes \kappa^{2}\right) u\right)$.

Let us notice that the operator $F$ (cf. Theorem 5.4) associated with the fundamental representation $u$ is given by the formula

$$
F_{u}=\left(\begin{array}{cc}
|\mu|^{-1}, & 0 \\
0, & |\mu|
\end{array}\right) \text {. }
$$

Indeed $\operatorname{Tr} F_{u}=\operatorname{Tr} F_{u}^{-1}=\left|\mu+\mu^{-1}\right|>0$ and the elementary computation shows that $\left(F_{u} \otimes I\right) u=u^{c c}\left(F_{u} \otimes I\right)$ [the latter means that $\left.F_{u} \in \operatorname{Mor}\left(u, u^{c c}\right)\right]$.

Using (5.22) one can compute the functionals (5.21) for $S_{\mu} U(2)$. We have

$$
\begin{gathered}
f_{z}(\alpha)=|\mu|^{-z}, \quad f_{z}\left(\gamma^{*}\right)=0, \\
f_{z}(\gamma)=0, \quad f_{z}\left(\alpha^{*}\right)=|\mu|^{z} .
\end{gathered}
$$


Now taking into account (A 1.1) we easily obtain the following convolution product formulae

$$
\begin{array}{cc}
f_{z} * \alpha=|\mu|^{-z} \alpha, & f_{z} * \gamma^{*}=|\mu|^{z} \gamma^{*}, \\
f_{z} * \gamma=|\mu|^{-z} \gamma, & f_{z} * \alpha^{*}=|\mu|^{z} \alpha^{*}, \\
\alpha * f_{z}=|\mu|^{-z} \alpha, & \gamma^{*} * f_{z}=|\mu|^{-z} \gamma^{*}, \\
\gamma * f_{z}=|\mu|^{z} \gamma, & \alpha^{*} * f_{z}=|\mu|^{z} \alpha^{*} .
\end{array}
$$

In particular $f_{1} * \alpha * f_{1}=\mu^{-2} \alpha$. Therefore [cf. (5.20)]

$$
h(\alpha b)=\mu^{-2} h(b \alpha)
$$

for any $b \in A$.

For any $k=\ldots,-2,-1,0,1,2, \ldots ; n, m=0,1,2, \ldots$, we set

$$
a_{k m n}= \begin{cases}\alpha^{k} \gamma^{* m} \gamma^{n} & \text { for } k \geqq 0 \\ \left(\alpha^{*}\right)^{-k} \gamma^{* m} \gamma^{n} & \text { for } k<1 .\end{cases}
$$

It is known that the family of all elements (A 1.5) forms a basis in the vector space $\mathscr{A}$ (cf. [18, Theorem 1.2]). Taking into account (A 1.3) and remembering that functionals $f_{z}(z \in \mathbb{C})$ are multiplicative, we get

$$
\begin{aligned}
& f_{z} * a_{k m n}=|\mu|^{(-k-n+m) z} a_{k m n}, \\
& a_{k m n} * f_{z}=|\mu|^{(-k+n-m) z} a_{k m n},
\end{aligned}
$$

for all $k, m, n$. On the other hand $f_{z}(I)=1$. Therefore according to (4.19) $f_{z} * h$ $=h * f_{z}=h$, and using (1.51) we see that $h\left(a * f_{z}\right)=h\left(f_{z} * a\right)=h(a)$ for any $a \in \mathscr{A}$. Combining this result with (A 1.6) and (A 1.7) we get

$$
h\left(a_{k m n}\right)=0
$$

for all $k, m, n$ except the cases when $k=0$ and $m=n$. Let $b_{m}=a_{0 m m}=\left(\gamma^{*} \gamma\right)^{m}$, where $m=0,1,2, \ldots$ In order to compute the remaining value of (A 1.8) we set $b=\alpha^{*} b_{m}$ in (A 1.4):

$$
h\left(\alpha \alpha^{*} b_{m}\right)=\mu^{-2} h\left(\alpha^{*} b_{m} \alpha\right) .
$$

Using commutation relations (1.33) one can easily check that $\alpha \alpha^{*} b_{m}=b_{m}-\mu^{2} b_{m+1}$ and $\alpha^{*} b_{m} \alpha=\mu^{-2 m}\left(b_{m}-b_{m+1}\right)$. Inserting these data into the above formula we get after simple transformations

$$
\frac{h\left(b_{m+1}\right)}{h\left(b_{m}\right)}=\frac{\left(1-\mu^{2 m+4}\right)^{-1}}{\left(1-\mu^{2 m+2}\right)^{-1}} .
$$

Therefore

$$
h\left(b_{m}\right)=\frac{\text { const }}{\left(1-\mu^{2 m+2}\right)},
$$

where the constant can be computed by setting $m=0: b_{0}=I, h(I)=1$. Hence const $=1-\mu^{2}$. Finally we have

$$
h\left(\left(\gamma^{*} \gamma\right)^{m}\right)=\frac{1-\mu^{2}}{\left(1-\mu^{2 m+2}\right)}
$$


for $m=0,1,2, \ldots$. Solving the corresponding problem of moments we get for any function $X$ continuous on the spectrum of $\gamma^{*} \gamma$,

$$
h\left(X\left(\gamma^{*} \gamma\right)\right)=N^{-1} \sum_{k=0}^{\infty} \mu^{2 k} X\left(\mu^{2 k}\right),
$$

where $N=\sum_{k=0}^{\infty} \mu^{2 k}=\left(1-\mu^{2}\right)^{-1}$ is the normalization factor.

Let $H$ be a Hilbert space with an orthonormal basis $\left(\psi_{n k}: n=0,1,2, \ldots\right.$; $k$-integer) and $\pi$ be the representation of the $C^{*}$-algebra $A$ acting on $H$ such that (cf. [18]):

$$
\begin{aligned}
& \pi(\alpha) \psi_{n k}=\sqrt{1-\mu^{2 n}} \psi_{n-1, k}, \\
& \pi(\gamma) \psi_{n k}=\mu^{n} \psi_{n, k+1} .
\end{aligned}
$$

We claim that

$$
h(a)=N^{-1} \sum_{n=0}^{\infty} \mu^{2 n}\left(\psi_{n 0} \mid \pi(a) \psi_{n 0}\right)
$$

for any $a \in A$. One can prove this formula by checking that it gives the right values for $a=a_{k m n}$. The details are left to the reader.

Let $d_{n}$ be the $(2 n+1)$-dimensional representation of $S_{\mu} U(2)$. We shall compute the character $\chi_{n}$ of $d_{n}$. For $n=0, d_{n}$ is the one-dimensional trivial representation and $\chi_{0}=I$. For $n=\frac{1}{2}, d_{1 / 2}$ is the fundamental representation and [cf. (1.34)] $\chi_{1 / 2}=\alpha+\alpha^{*}$. Let $n \geqq \frac{1}{2}$. We know (cf. [18, Theorem 5.11]) that $d_{n} \oplus d_{1 / 2}$ is equivalent to the direct sum $d_{n-1 / 2} \oplus d_{n+1 / 2}$. Therefore

$$
\chi_{n+1 / 2}=\chi_{n} \chi_{1 / 2}-\chi_{n-1 / 2} \text {. }
$$

Solving this recursive equation we get

$$
\chi_{n}=\frac{\sin \left(\frac{2 n+1}{2} t\right)}{\sin \left(\frac{1}{2} t\right)},
$$

where $t=2 \arccos \left(\frac{\alpha+\alpha^{*}}{2}\right)$.

Let us notice that the functions (A 1.9) form an orthonormal sequence on the interval $[0,2 \pi]$ with respect to the measure $\frac{1}{\pi}\left(\sin _{2} \frac{1}{2} t\right)^{2} d t$. Therefore for any
$Y \in C($ Spectrum $t)$,

$$
h(Y(t))=\frac{1}{\pi} \int_{0}^{2 \pi} Y(\tau)\left(\sin \frac{1}{2} \tau\right)^{2} d \tau .
$$

Taking into account the definition of $t$ and substituting $x=\cos \frac{1}{2} \tau$ we get

$$
h\left(Z\left(\frac{\alpha+\alpha^{*}}{2}\right)\right)=\frac{2}{\pi} \int_{-1}^{1} Z(x) \sqrt{1-x^{2}} d x
$$

for any $Z \in C([-1,1])$. 


\section{A 2. Finite Matrix Pseudogroups}

Let $G=(A, u)$ be a compact matrix pseudogroup. We say that $G$ is finite if $\operatorname{dim} A<\infty$. In this Appendix we show that many formulae derived in this paper may be essentially simplified if the considered pseudogroup is finite. In particular we find the simple formula defining the Haar measure on finite matrix pseudogroups.

Any finite-dimensional $C^{*}$-algebra is a direct sum of full matrix algebras. Having this fact in mind one can easily prove

Proposition A 2.1. Let $A$ be a $C^{*}$-algebra and $Z(A)$ be the center of $A$ :

$$
Z(A)=\{a \in A: a b=b a \text { for any } b \in A\} .
$$

Assume that $A$ is finite-dimensional. Then

1. For any linear multiplicative functional $f$ defined on $A$ there exists a unique minimal projection $f_{A} \in A$ such that $f_{A} a=a f_{A}=f(a) f_{A}$. In particular the number of linear multiplicative functionals on $A$ is finite.

2. There exists a unique positive linear functional $\operatorname{Tr}$ on $A$ such that $\operatorname{Tr} P=1$ for any minimal projection $P \in A$. This functional is central: $\operatorname{Tr}(a b)=\operatorname{Tr}(b a)$ for any $a, b \in A$.

3. Any central linear functional $\tau$ on $A$ is of the form

$$
\tau(a)=\sum_{P \in C} c_{P} \operatorname{Tr}(P a)
$$

where $C$ is the set of all minimal projections in $Z(A)$ and $c_{P}(P \in C)$ are complex constants.

4.

$$
\operatorname{dim} A=\sum_{P \in C}(\operatorname{Tr} P)^{2} .
$$

Let $G=(A, u)$ be a finite matrix pseudogroup. Then the dense *-subalgebra $\mathscr{A}$ generated by matrix elements of $u$ coincides with $A$ and the functionals $e$ and $f_{z}$ introduced in Proposition 1.8 and Theorem 5.6 are defined on the whole $A$. It follows immediately from Proposition A 2.1.1 that $f_{z}$ does not depend on $z$. Therefore $f_{z}=f_{0}=e$ for any $z \in \mathbb{C}$. This fact simplifies many formulae of Sect. 5. In particular using (1.52), Theorem 5.6.5 and 6 taking into account (1.4) we obtain

Proposition A 2.2. 1. The Haar measure $h$ on $G$ is central: $h(a b)=h(b a)$ for any $a, b \in A$.

2. The coinverse $\kappa$ associated with $G$ is an involutive $C^{*}$-antiisomorphism of $A$ : $\kappa(\kappa(a))=a$ and $\kappa\left(a^{*}\right)=\kappa(a)^{*}$ for any $a \in A$.

In particular $\operatorname{Tr}$ is $\kappa$-invariant:

$$
\operatorname{Tr}(\kappa(a))=\operatorname{Tr}(a)
$$

for any $a \in A$.

Let $\Phi$ be the comultiplication associated with $G$ and $e_{A} \in A$ be the minimal projection corresponding to the linear multiplicative functional $e$ (cf. Proposition A 2.1.1)

$$
e_{A} a=a e_{A}=e(a) e_{A}
$$


for any $a \in A$. We shall prove that

$$
(I \otimes a) \Phi\left(e_{A}\right)=(\kappa(a) \otimes I) \Phi\left(e_{A}\right)
$$

for any $a \in A$. Indeed by virtue of Theorem 4.9 one may find $b_{m}, c_{m} \in A$ $(m=1,2, \ldots, M)$ such that

$$
\begin{aligned}
I \otimes a & =\sum_{m}\left(b_{m} \otimes I\right) \Phi\left(c_{m}\right), \\
I \otimes \kappa(a) & =\sum_{m} \Phi\left(b_{m}\right)\left(c_{m} \otimes I\right) .
\end{aligned}
$$

Applying $(e \otimes \mathrm{id})$ to both sides of (A 2.7) and using (1.52) we get

$$
\kappa(a)=\sum_{m} b_{m} e\left(c_{m}\right) .
$$

Now (A 2.5) can be checked by direct computation. Using (A 2.6), (A 2.4) and the last formula we have

$$
(I \otimes a) \Phi\left(e_{A}\right)=\sum_{m}\left(b_{m} \otimes I\right) \Phi\left(c_{m} e_{A}\right)=\sum_{m}\left(b_{m} \otimes I\right) e\left(c_{m}\right) \Phi\left(e_{A}\right)=(\kappa(a) \otimes I) \Phi\left(e_{A}\right) .
$$

Let $R$ be the minimal projection in $Z(A)$ (center of $A$ ) and $E_{R}=(R \otimes I) \Phi\left(e_{A}\right)$. Clearly $E_{R}$ is a projection and using Theorem 4.9 we see that $E_{R} \neq 0$.

Let $Q$ be a minimal projection in $A \otimes A$ such that $Q \leqq E_{R}$. Then $Q \leqq \Phi\left(e_{A}\right)$, $Q=\Phi\left(e_{A}\right) Q$, and using (A 2.5) we have

$$
(I \otimes a) Q=(\kappa(a) \otimes I) Q
$$

for any $a \in A$. Computing the hermitian conjugation of both sides of this relation, replacing $a^{*}$ by $a$ and taking into account Proposition A 2.2.2 we get

$$
Q(I \otimes a)=Q(\kappa(a) \otimes I)
$$

for any $a \in A$. Let $a, b \in A$. Using the last two formulae we have

$$
\begin{gathered}
Q(I \otimes b a) Q=Q(I \otimes b)(I \otimes a) Q=Q(I \otimes b)(\kappa(a) \otimes I) Q \\
=Q(\kappa(a) \otimes I)(I \otimes b) Q=Q(I \otimes a)(I \otimes b) Q=Q(I \otimes a b) Q .
\end{gathered}
$$

This relation shows that the linear functional

$$
A \ni a \mapsto(\operatorname{Tr} \otimes \operatorname{Tr})((I \otimes a) Q)
$$

is central. Therefore (cf. Proposition A 2.1.3)

$$
(\operatorname{Tr} \otimes \operatorname{Tr})((I \otimes a) Q)=\sum_{P \in C} c_{P} \operatorname{Tr}(a P),
$$

where $C$ is the set of all minimal projections in $Z(A)$ and the constants $c_{P}(P \in C)$ are independent of $a \in A$.

We know that $Q \leqq E_{R} \leqq R \otimes I$. Therefore $(R \otimes I) Q=Q$, and using (A 2.8) we have $(I \otimes \kappa(R)) Q=Q\left(\kappa^{-1}=\kappa\right)$. Replacing in (A 2.10) $a$ by $a \kappa(R)$, we get

$$
(\operatorname{Tr} \otimes \operatorname{Tr})((I \otimes a) Q)=c_{\kappa(R)} \operatorname{Tr}(a \kappa(R)) .
$$

We know that $Q$ is a minimal projection in $A \otimes A$. Therefore $(\operatorname{Tr} \otimes \operatorname{Tr})(Q)=1$ and inserting in (A 2.11) $a=I$ we see that $c_{\kappa(R)} \operatorname{Tr}(\kappa(R))=1$ and [cf. (A 2.3)] $c_{\kappa(R)}$ $=(\operatorname{Tr} R)^{-1}$. 
Now using (A 2.8) and (A 2.11) we obtain

$$
\begin{aligned}
(\operatorname{Tr} \otimes \operatorname{Tr})((b \otimes a) Q) & =(\operatorname{Tr} \otimes \operatorname{Tr})((I \otimes a \kappa(b)) Q) \\
& =\frac{\operatorname{Tr}(a \kappa(b) \kappa(R))}{\operatorname{Tr} R}
\end{aligned}
$$

for any $a, b \in A$. Clearly this formula determines $Q$ uniquely. Therefore there exists only one minimal projection in $A \otimes A$ majorized by $E_{R}$. In other words $E_{R}$ is minimal, $Q=E_{R}=(R \otimes I) \Phi\left(e_{A}\right)$ and

$$
(\operatorname{Tr} \otimes \operatorname{Tr})\left((b R \otimes a) \Phi\left(e_{A}\right)\right)=\frac{\operatorname{Tr}(a \kappa(b) \kappa(R))}{\operatorname{Tr} R} .
$$

Multiplying both sides by $\operatorname{Tr} R$ and summing over $R \in C$ we get

$$
(\operatorname{Tr} \otimes \operatorname{Tr})\left((b T \otimes a) \Phi\left(e_{A}\right)\right)=\operatorname{Tr}(a \kappa(b)),
$$

where

$$
T=\sum_{R \in C}(\operatorname{Tr} R) R
$$

and $a, b \in A$. Using the freedom of $a$ we obtain

$$
(\operatorname{Tr} \otimes \mathrm{id})\left((b T \otimes I) \Phi\left(e_{A}\right)\right)=\kappa(b) .
$$

Let $h$ be the Haar measure on $G$. Applying $h$ to both sides of the above relation and using (4.21) we get

$$
h(b)=(\operatorname{Tr} \otimes h)\left((b T \otimes I) \Phi\left(e_{A}\right)\right) .
$$

On the other hand according to (4.20) (id $\otimes h) \Phi\left(e_{A}\right)=h * e_{A}=h\left(e_{A}\right) I$ and

$$
h(b)=\operatorname{Tr}(b T) h\left(e_{A}\right) \text {. }
$$

By virtue of (A 2.2) $\operatorname{Tr} T=\sum_{R \in C}(\operatorname{Tr} R)^{2}=\operatorname{dim} A$. Therefore the normalization $h(I)=1$ implies that $h\left(e_{A}\right)=\frac{1}{\operatorname{dim} A}$ and finally we have

$$
h(b)=\frac{1}{\operatorname{dim} A} \operatorname{Tr}(b T)
$$

where $b \in A$ and $T$ is given by (A 2.12).

\section{References}

1. Barut, A.O., Raczka, R.: Theory of group representations and applications. Warszawa: PWN - Polish Scientific Publishers 1977

2. Bragiel, K.: Twisted $S U(3)$ group (in preparation)

3. Dixmier, J.: Les $C^{*}$-algèbres et leurs representations. Paris: Gauthier, Villars 1964

4. Drinfeld, V.S.: Quantum groups, will appear in Proceedings ICM - 1986

5. Enock, M., Schwartz, J.M.: Une dualité dans les algèbres de von Neumann. Bull. Soc. Math. France, Suplément mémoire 44, 1-144 (1975)

Schwartz, J.M.: Sur la structure des algèbres des Kac I. J. Funct. Anal. 34, $370-406$ (1979) 
6. Kac, G.I.: Ring-groups and the principle of duality I and II. Trudy Moskov. Mat. Obsc. 12, 259-301 (1963); 13, 84-113 (1965)

7. Kruszynski, P., Woronowicz, S.L.: A noncommutative Gelfand-Naimark theorem. J. Oper. Theory 8, 361-389 (1982)

8. Lang, S.: Algebra. Reading, MA: Addison-Wesley 1965

9. Maurin, K.: Analysis I. Warsaw-Dordrecht: PWN - Polish Scientific Publishers, Dordrecht: Reidel 1976

10. Ocneanu, A.: A Galois theory for operator algebras. Preprint

11. Takesaki, M.: Duality and von Neumann algebras. Lecture notes, Fall 1970, Tulane University, New Orleans, Louisiana

12. Tatsuuma, N.: An extension of AKHT theory of locally compact groups. Kokyuroku RIMS, 314 (1977)

13. Vallin, J.M.: $C^{*}$-algèbres de Hopf et $C^{*}$-algèbres de Kac. Proc. Lond. Math. Soc. (3), 50, 131-174 (1985)

14. Vaksman, L.L., Soibelman, J.S.: The algebra of functions on quantum group $S U(2)$ (to appear)

15. Weyl, H.: The classical groups, their invariants and representations. Princeton, NS: Princeton University Press 1946

16. Woronowicz, S.L.: On the purification of factor states. Commun. Math. Phys. 28, 221-235 (1972)

17. Woronowicz, S.L.: Pseudospaces, pseudogroups, and Pontryagin duality. Proceedings of the International Conference on Mathematics and Physics, Lausanne 1979. Lecture Notes in Physics, Vol. 116. Berlin, Heidelberg, New York: Springer 1980

18. Woronowicz, S.L.: Twisted $S U(2)$ group. An example of a non-commutative differential calculus, will appear in RIMS - Publ. University of Kyoto (1987)

19. Woronowicz, S.L.: Tannaka-Krein duality for compact matrix pseudogroups. Twisted $S U(N)$ groups (in preparation)

20. Woronowicz, S.L.: Differential calculus on compact matrix pseudogroups (in preparation)

Communicated by H. Araki

Received January 8, 1987; in revised form March 27, 1987 
